\title{
Statistical Stellar Mass Corrections for High-z Galaxies Observed with JWST Broadband Filters Due to Template Degeneracies
}

\author{
L. Bisigello ${ }^{1,2}$ (D) , K. I. Caputi ${ }^{1,3}$ (D) , L. Colina ${ }^{3,4}$, P. G. Pérez-González ${ }^{4,5,6}$ (D), A. Koekemoer ${ }^{7}$ (D) O. Le Fèvre ${ }^{8}$, N. Grogin ${ }^{7}$ (D), \\ H. U. Nørgaard-Nielsen ${ }^{9}$, and P. van der Werf $^{10}$ (D) \\ ${ }^{1}$ Kapteyn Astronomical Institute, University of Groningen, P.O. Box 800, $9700 \mathrm{AV}$, Groningen, The Netherlands \\ ${ }^{2}$ University of Nottingham, School of Physics \& Astronomy, Nottingham, NG7 2RD, UK \\ ${ }^{3}$ Cosmic Dawn Center (DAWN), Niels Bohr Institute, University of Copenhagen, Juliane Maries vej 30, DK-2100 Copenhagen, Denmark \\ ${ }^{4}$ Centro de Astrobiología, Departamento de Astrofísica, CSIC-INTA, Cra. de Ajalvir km.4, E-28850—Torrejón de Ardoz, Madrid, Spain \\ ${ }_{5}^{5}$ Departamento de Astrofísica, Facultad de CC. Físicas, Universidad Complutense de Madrid, E-28040 Madrid, Spain \\ ${ }^{6}$ Associate Astronomer at Steward Observatory, The University of Arizona, USA \\ ${ }^{7}$ Space Telescope Science Institute, 3700 San Martin Drive, Baltimore, MD 21218, USA \\ ${ }^{8}$ Aix Marseille Université, CNRS, LAM (Laboratoire d'Astrophysique de Marseille), UMR 7326, F-13388, Marseille, France \\ ${ }^{9}$ National Space Institute (DTU Space), Technical University of Denmark, Elektrovej, DK-2800 Kgs. Lyngby, Denmark \\ ${ }^{10}$ Sterrewacht Leiden, Leiden University, P.O. Box 9513, 2300 RA, Leiden, The Netherlands \\ Received 2018 October 4; revised 2019 May 24; accepted 2019 June 10; published 2019 August 1
}

\begin{abstract}
Stellar masses in future James Webb Space Telescope (JWST) deep blank fields will be mainly derived by fitting the spectral energy distribution (SED) with theoretical galaxy templates. We investigate the uncertainties and biases of the stellar masses derived by using the LePhare code for SED fitting and the Yggdrasil theoretical templates. We consider a sample of mock galaxies at $z=7-10$ with mock JWST observations with $\mathrm{S} / \mathrm{N}_{\mathrm{F} 150 \mathrm{~W}} \geqslant 10$. Our goal is to provide a list of statistical stellar mass corrections to include on the stellar mass derivation for different output galaxy properties and JWST filter combinations to correct for template degeneracies. Median statistical stellar mass corrections vary from -0.83 to 0.87 dex, while $25 \%$ (75\%) quartiles range from $-0.83(-0.67)$ to $0.51(0.88)$ dex, depending on filter combinations and galaxy models. The most challenging cases are galaxies with nebular emission lines, especially the ones that are wrongly identified as galaxies without, relative dust-free galaxies, and galaxies with small metallicities (i.e., $Z=1 / 50 Z_{\odot}$ ). The stellar mass estimation of galaxies correctly identified without emission lines is generally fine, except at $z=10$ when considering only the eight NIRCam bands, which make the MIRI bands very valuable. We have tested our stellar mass corrections using the public JAGUAR galaxy catalog, deriving that the average discrepancy in the recovered stellar mass distribution decreases by $20 \%-50 \%$ at $z>7$ after the correction. We found that without the stellar mass corrections, the number of low-mass galaxies $\left(M^{*}<10^{7} M_{\odot}\right)$ is overestimated, which can potentially lead to systematic errors in the calculation of the galaxy stellar mass function faint-end slope at high $z$.
\end{abstract}

Key words: galaxies: fundamental parameters - galaxies: high-redshift - galaxies: photometry

Supporting material: machine-readable tables

\section{Introduction}

Stellar mass is one of the most fundamental properties of galaxies, as it has a central role in galaxy evolution (Peng et al. 2010). Its importance is evident from the numerous relations that are present between galaxy stellar mass and other galaxy properties, such as star formation rate (e.g., Brinchmann et al. 2004; Noeske et al. 2007; Rodighiero et al. 2011; Whitaker et al. 2014; Tasca et al. 2015; Bisigello et al. 2018; Boogaard et al. 2018) and metallicity (e.g., Tremonti et al. 2004; Erb et al. 2006; Maiolino et al. 2008; Maier et al. 2015).

By fitting the spectral energy distribution (SED) of galaxies derived from broadband photometry with theoretical or empirical templates, it is possible to derive a broad set of galaxy properties, among which there is also the stellar mass. This is a powerful technique because it allows for deriving properties of galaxies up to high redshift, thanks to the possibility of obtaining broadband photometry for large samples of faint galaxies. Comparatively, with spectroscopy, it is possible to have a more reliable estimate of some of these galaxy properties, but spectroscopic observations require more integration time than photometry and are usually possible only for relatively bright galaxies.
It has been shown in previous works that, once the redshift of a galaxy is well known, the stellar mass is one of the most robust parameters derived from the SED fitting (e.g., Caputi et al. 2015). However, precise stellar masses are particularly difficult to estimate in some situations. Indeed, galaxies with ongoing strong episodes of star formation are dominated by a young stellar population, and the stellar mass derivation is strongly affected by uncertainties in the age estimation. In addition, these young and star-forming galaxies may have numerous nebular emission lines with high equivalent widths that contaminate the broadband observations and affect the derived stellar mass (e.g., Stark et al. 2013; Santini et al. 2015; Caputi et al. 2017). These nebular emission lines are generally visible as a flux excess in some bands, but in some extreme cases, they are so numerous as to boost the flux of contiguous bands and mimic a higher continuum. This results in a degeneracy between young galaxies with numerous emission lines and more massive, older galaxies (Bisigello et al. 2017).

In addition, observations at the rest-frame near-ultraviolet are affected by dust extinction, and the mass-to-light ratio at these wavelengths is sensitive to small differences in the stellar population age. Therefore, it is necessary to have observations at wavelengths longer than $4000 \AA$ to have a good estimate of 
the stellar mass. This means that at very high-z, it is necessary to observe at near-IR wavelengths to have a good stellar mass estimation, and this will indeed be possible in the near future with the James Webb Space Telescope (JWST; ${ }^{11}$ Gardner et al. 2009).

The JWST is among the most promising facilities of upcoming years. It has a $6.5 \mathrm{~m}$ primary mirror and four instruments on board observing at near- and mid-IR wavelengths $(0.6-28 \mu \mathrm{m})$ with imaging, spectroscopic, and coronographic modes. In particular, the Near Infrared Camera (NIRCam; Rieke et al. 2005) is an imaging camera covering $0.6-5 \mu \mathrm{m}$ with different broad-, intermediate-, and narrowband filters. On the other hand, the complementary Mid Infrared Instrument (MIRI; Rieke et al. 2015; Wright et al. 2015) has nine broadband filters that cover between 5 and $28 \mu \mathrm{m}$. These two imaging cameras will be the main instruments used to carry out deep blank-field imaging surveys with JWST to detect high$z$ galaxies.

The properties of galaxies observed in these deep blank-field imaging surveys will be mainly derived using SED fitting. In Bisigello et al. (2016, 2017; hereafter B16 and B17, respectively), we created and analyzed a sample of mock galaxies at $z=7-10$ to study how different galaxy properties will be derived using SED fitting and different NIRCam and MIRI broadband filter combinations. In particular, in B17, we show that stellar masses may be particularly difficult to estimate for specific filter combinations and particular galaxy templates, i.e., galaxies with numerous nebular emission lines and at $z=10$, in particular if mid-IR observations are not available. The aim of this paper is to derive and analyze the stellar mass offset for each specific galaxy template and for different JWST broadband filter combinations to compensate for template degeneracies or lack of wavelength coverage. These corrections will be extremely useful to statistically correct the stellar mass of a large sample of high- $z$ galaxies that will be observed using different combinations of JWST broadband filters in the near future to study, for example, the stellar mass function at $z>7$.

The paper is structured as follows. In Section 2 we describe the analyzed sample of mock galaxies, the photometry extraction in the pertinent NIRCam and MIRI bands, and the stellar mass derivation. We present our stellar mass corrections for different JWST broadband filter combinations in Section 3. In addition, we analyze the stellar mass correction with respect to other galaxy properties, such as redshift, age, color excess, metallicity, and star formation history (SFH). In Section 4 we give practical information on how to include the derived stellar mass correction in future studies, and in Section 5 we apply and test the derived corrections to a galaxy sample. Finally, in Section 6 we summarize our main findings and conclusions. Throughout this paper, we consider a cosmology with $H_{0}=$ $70 \mathrm{~km} \mathrm{~s}^{-1} \mathrm{Mpc}^{-1}, \Omega_{M}=0.27, \Omega_{\Lambda}=0.73$, and a Kroupa (2002) initial mass function (IMF). All magnitudes refer to the AB system (Oke \& Gunn 1983).

\section{Sample}

\subsection{Sample Selection}

Our study is based on a sample of 750 simulated galaxies at $z=7-10$, presented in B16 and derived from the Yggdrasil population synthesis code (Zackrisson et al. 2011), which is

\footnotetext{
11 http://www.jwst.nasa.gov
}

specifically built to describe high- $z$ galaxies. These templates have solar and subsolar metallicities, step function SFHs, and a color excess between zero and 0.25 mag considering a Calzetti et al. (2000) reddening law. We apply the same dust attenuation for continuum and nebular emission lines; however, considering different dust extinction values for the emission lines and continuum may vary some of the template degeneracies but does not create any systematic shift in the statistical stellar mass correction. Ages are between 0.01 and $0.6 \mathrm{Gyr}$ and consistent with the age of the universe at $z=7-10$, and we consider a Kroupa (2002) IMF. The nebular continuum and emission lines are already incorporated in the Yggdrasil templates when the galaxy is star-forming and the covering factor is not zero. We consider two different covering factors, corresponding to galaxies without nebular emission lines, $f_{\text {cov }}=0$, and with the maximum contribution from the nebular lines, $f_{\text {cov }}=1$. These values correspond to a Lyman continuum escape fraction of 1 and zero, respectively, as $f_{\text {esc }}=1-f_{\text {cov }}$.

\subsection{Mock JWST Photometry}

For all 750 templates, we have mock observations for the eight NIRCam broad bands and the two MIRI broad bands F560W and F770W, as explained in detail in B16. These mock observations are obtained by convolving each template with the corresponding JWST filter. All simulated galaxies are normalized at $29 \mathrm{AB}$ mag at $1.5 \mu \mathrm{m}$, which corresponds to the pivot wavelength of the F150W NIRCam band. Signal-to-noise (S/N) values of 10 and 20 are considered for the F150W band, and the same integration time is assumed for all other NIRCam bands. For the MIRI bands, we consider the same $\mathrm{S} / \mathrm{N}$ as the F150W NIRCam band but for a magnitude brighter, i.e., 28 AB mag. This has been done to take into account different sensitivities between the NIRCam and MIRI bands due to different detector technologies. We consider only an $\mathrm{S} / \mathrm{N} \geqslant 10$, because for more than $99 \%$ of the simulated galaxies at $z=7-10$ with an $\mathrm{S} / \mathrm{N}$ of 10 , the photometric redshift is already well recovered, i.e., $\mid z_{\text {phot }}-z_{\text {fiducial }} /\left(1+z_{\text {fiducial }}\right) \leqslant 0.15$, using only eight NIRCam bands. Therefore, errors in the stellar masses are only due to degeneracies between templates and not to a drastically incorrect redshift estimation. For each simulated galaxy, we have 100 mock observations in the considered NIRCam and MIRI bands, derived by randomizing each flux inside the error bars, for a total sample of 75,000 mock observations. The results presented in this paper are also valid for galaxies with stellar masses different from the analyzed ones, i.e., corresponding to $29 \mathrm{AB}$ mag at $1.5 \mu \mathrm{m}$, as long as the $\mathrm{S} / \mathrm{N} \geqslant 10$ and the SED shapes are the same. In general, it is necessary to consider with caution the application of these results to stellar masses derived by using templates with extremely different prescriptions for nebular emission lines, SFHs, or the general SED shape. In addition, it is necessary to take into account that the stellar mass offsets analyzed in this work are due only to template degeneracies and are therefore nonexhaustive, as, for example, additional errors are expected due to differences between idealized theoretical templates and real galaxy SEDs.

\subsection{Galaxy Properties Derivation}

The redshift and stellar mass recovery for these simulated galaxies have been derived and analyzed in B16 and B17, respectively. In particular, galaxy properties have been 
obtained for different combinations of JWST broadband observations:

1. eight NIRCam broad bands,

2. eight NIRCam broad bands and two MIRI bands (F560W and F770W),

3. eight NIRCam broad bands and MIRI F560W only, and

4. eight NIRCam broad bands and MIRI F770W only.

For all of these different filter combinations, we derive the stellar mass and photometric redshift using the public code LePhare (Arnouts et al. 1999; Ilbert et al. 2006) and considering a large set of possible output templates, including the ones used to derive the mock observations. In particular, we consider a wide range of color excess, i.e., from zero to $1 \mathrm{mag}$ with a step $0.05 \mathrm{mag}$; redshifts from zero to 11 ; and ages from 0.01 to $5 \mathrm{Gyr}$ and consistent with the age of the universe. We also include Bruzual \& Charlot (2003) templates with exponentially declining SFHs with the same values for redshift, age, and color excess as the Yggdrasil templates and with emission lines. However, almost all galaxies are best fit by Yggdrasil, and the very few exceptions result in extremely large redshift errors, i.e., output $z<2$. We include two covering factors, zero and 1 , but only when the star formation is ongoing, as this parameter does not influence the SED when the galaxy is not star-forming. For more details on the set of template parameters used to derive the stellar mass and the procedure to estimate it, we refer to B17.

In this paper, we present the stellar mass correction for galaxies corresponding to each combination of output properties. We limit our analyses to mock galaxies with a combination of output parameters that are present among the input ones but not necessarily the correct one. The stellar mass correction for galaxies with output parameter values not present among the input ones would be overestimated, because the output galaxy model is always different from the input one by construction, and, therefore, the stellar mass is never correct. We divide color excesses and redshifts in bins of $\Delta(E(B-V))=0.1 \mathrm{mag}$ and $\Delta z=1$ centered around the values present among the mock observations.

Overall, we remove from the sample galaxies with output redshifts below 6.5 and above 10.5 , which correspond to $<1.2 \%$ and $<2.3 \%$ with all band combinations. Moreover, we do not consider galaxies with output color excess larger than 0.3 mag that correspond to $1.3 \%$ of the sample when only NIRCam broad bands are considered and $<0.5 \%$ when at least one of the two MIRI bands is included. The full set of galaxy properties considered in this work is listed in Table 1, and their input distributions are shown in Figure 1. Given these points, the final samples consist of 71,257 (95\% of the original catalog) galaxies with NIRCam observations, 72,897 (97\% of the original catalog) galaxies with NIRCam and F560W band observations, 72,295 (96\% of the original catalog) galaxies with NIRCam and F770W band observations, and 73,741 (98\% of the original catalog) galaxies with observations in the eight NIRCam and two MIRI bands.

The stellar mass of each mock galaxy is obtained by LePhare by scaling the template considering all bands, while the input templates are scaled to match a magnitude $29 \mathrm{AB}$ at $1.5 \mu \mathrm{m}$. This difference may result in a bias on the derived stellar mass. Therefore, we analyze the recovered stellar mass for a subsample of mock galaxies for which all other galaxy
Table 1

Output Values of Different Galaxy Properties Considered in This Work

\begin{tabular}{lc}
\hline \hline Parameter & Values \\
\hline Metallicity & $Z_{\odot}, 0.4 Z_{\odot}, 0.2 Z_{\odot}, 0.02 Z_{\odot}{ }^{\mathrm{a}}$ \\
SFH type & Step function \\
SFH $(\mathrm{Gyr})$ & $0.01,0.03,0.1$ \\
$f_{\text {cov }}$ & $0,1^{\mathrm{b}}$ \\
$E(B-V)^{\mathrm{c}}$ & $0,0.05,0.1,0.15,0.2,0.25,0.3^{\mathrm{d}}$ \\
Age $(\mathrm{Gyr})$ & $0.01,0.05,0.2,0.4,0.6^{\mathrm{e}}$ \\
$z$ & $6.5,6.55,6.70, \ldots, 10.40,10.45,10.5^{\mathrm{f}}$
\end{tabular}

Notes.

${ }^{\mathrm{a}}$ For this metallicity, we consider only ages $t<0.2$ Gyr.

b Templates of old galaxies with no ongoing star formation do not change with the covering factor, so, for these galaxies, we consider only $f_{\text {cov }}=0$.

${ }^{\mathrm{c}}$ Following the Calzetti et al. reddening law (Calzetti et al. 2000).

${ }^{\mathrm{d}}$ Results are shown in bins of $\Delta(E(B-V))=0.1 \mathrm{mag}$.

e We consider this age only up to redshift $z=8$.

${ }^{\mathrm{f}}$ Results are shown in bins of $\Delta z=1$.

parameters are perfectly recovered; i.e., the input template is correctly recognized. We find that a small bias is present, and stellar masses are, on average, overestimated by $\sim 5 \%-6 \%$. Hereafter, all output stellar masses are corrected by this general bias of -0.025 dex to remove the dependence on the used SED fitting code.

\subsection{Dependence of the Stellar Mass Corrections on the Chosen Input Parameters}

The stellar mass corrections derived here depend on the assumed input galaxy population, which is derived assuming the parameters listed in Table 1 and following the distributions shown in Figure 1. All parameter combinations have been included, except for templates with ages longer than the universe's age at the considered redshift and the lowest metallicity $\left(0.02 Z_{\odot}\right)$, which is considered only for templates with ages shorter than $0.2 \mathrm{Gyr}$. The results presented in this paper are affected by the used parameters in two ways. First, templates described by different parameters may create additional degeneracies that are not taken into account here; therefore, the stellar mass corrections presented need to be considered as not exhaustive. Second, if the input distribution of each parameter is extremely different, the overall degeneracies will remain the same, but each probability may be different.

Under the assumption that nebular emission lines depend on metallicity, dust extinction, covering factor, age, and SFH, but not on stellar mass, the stellar mass corrections derived in this paper do not depend on the assumed stellar mass distribution. Indeed, if two templates well represent some observations, they will equally well represent the same observations scaled by an arbitrary factor, as long as the $\mathrm{S} / \mathrm{N}$ and scaling factor are the same for all observations. The absolute stellar mass would change because of the rescaling, but the stellar mass correction, which is a relative quantity, would remain the same. For this reason, our results do not depend on any assumption of the input stellar mass distribution and can be generally applied to any statistical sample. However, we advise against using the corrections presented here for samples biased on any of the other input parameters, i.e., a sample containing only emissionline galaxies. In the case of a biased sample, the corrections 


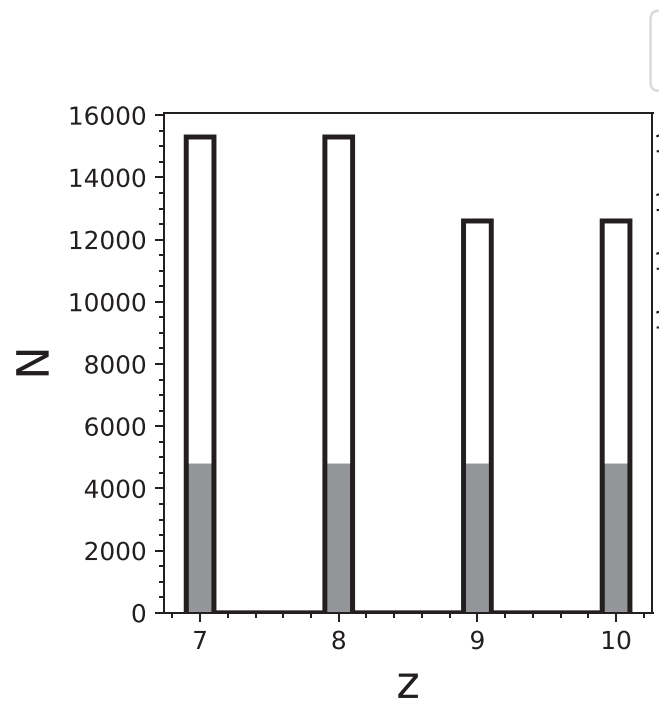

$\square \mathrm{f}_{\text {cov }}=0 \quad \mathrm{f}_{\text {cov }}=1$
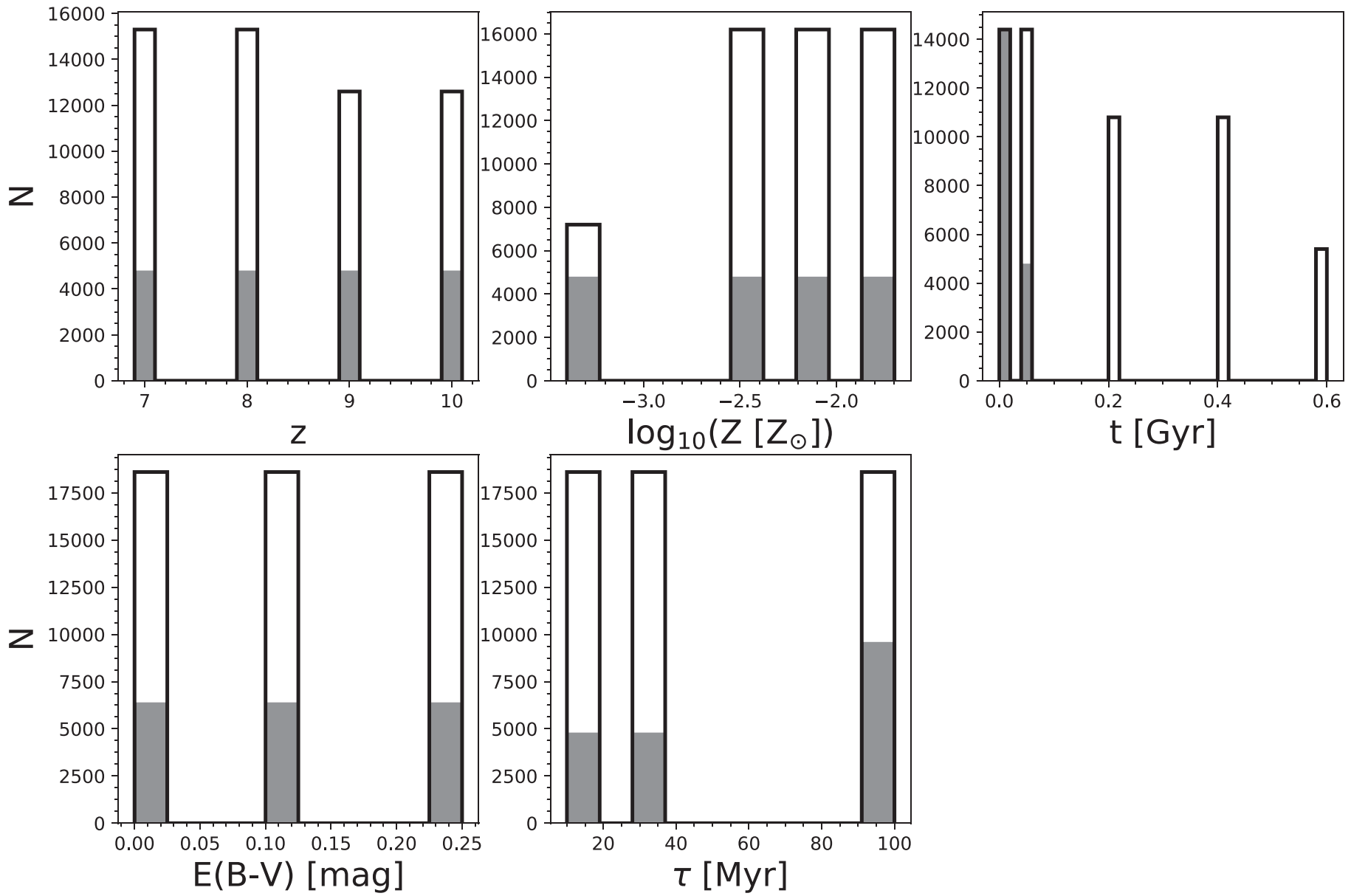

Figure 1. Distribution of each input galaxy parameter used to derive the galaxy sample considered in this work. From top left to bottom right: redshift, metallicity, galaxy age (time from the beginning of star formation), color excess, and duration of the star formation episodes. Each parameter distribution is shown separately for galaxies with $f_{\text {cov }}=0$ (open histograms) or $f_{\text {cov }}=1$ (filled histograms).

presented here can be considered to identify possible biases affecting the stellar mass.

\section{Results}

In this section, we present the statistical stellar mass corrections for the different JWST broadband filter combinations. These can be used to statistically correct the stellar mass estimate for degeneracies arising from the SED fitting procedure. Statistical stellar mass corrections are derived comparing the output stellar mass with the input one, i.e., $\log _{10}\left(M_{\text {out }}^{*}\right)-\log _{10}\left(M_{\text {in }}^{*}\right)$. The median statistical stellar mass corrections are in general small, as shown in Figures 2-6, but the quartile ranges are large; therefore, some galaxy models may need significant stellar mass corrections. In particular, the $25 \%$ quartiles range from -0.83 to 0.51 dex, while the $75 \%$ quartiles are between -0.51 and 0.88 dex.

In the next sections, we analyze how these stellar mass corrections are related to other output parameters, i.e., covering factor, redshift, age, color excess, SFH, and metallicity, to understand for which SED templates the stellar mass estimation is particularly challenging. All plots refer to mock galaxies with $\mathrm{S} / \mathrm{N}_{\mathrm{F} 150 \mathrm{~W}}=10$, but the results are similar for $\mathrm{S} / \mathrm{N}_{\mathrm{F} 150 \mathrm{~W}}=20$.
We include in the online material the detailed stellar mass offset distribution of both $\mathrm{S} / \mathrm{N}$ values.

\subsection{Variation of Statistical Stellar Mass Correction with Covering Factor}

We separate galaxies with output $f_{\text {cov }}=1$ or 0 , i.e., starforming galaxies with emission lines or galaxies without emission lines. Among all galaxies with output $f_{\text {cov }}=1,22 \%$ have input $f_{\text {cov }}=0$ when considering only the NIRCam bands. Among these galaxies, $83 \%(41 \%)$ have stellar mass offsets larger than $0.1(0.3)$ dex in modulus, with stellar masses that tend to be underestimated, even up to 10 times. Here we do not investigate the equivalent width of the nebular emission lines; therefore, galaxies there that are wrongly identified with $f_{\text {cov }}=1$ may have a very low output value of equivalent widths. On the other hand, even the population of galaxies correctly identified as galaxies with $f_{\text {cov }}=1$ shows large stellar mass offsets but with less frequency, i.e., $40 \%(22 \%)$ with stellar mass offsets larger than $0.1(0.3)$ dex in modulus. The inclusion of the MIRI bands decreases the percentage of galaxies with input $f_{\text {cov }}=0$ to $8 \%$ of all galaxies with output $f_{\text {cov }}=1$.

Among galaxies with output $f_{\text {cov }}=0, \sim 5 \%$ have input $f_{\text {cov }}=1$ when considering only NIRCam bands. This percentage only 


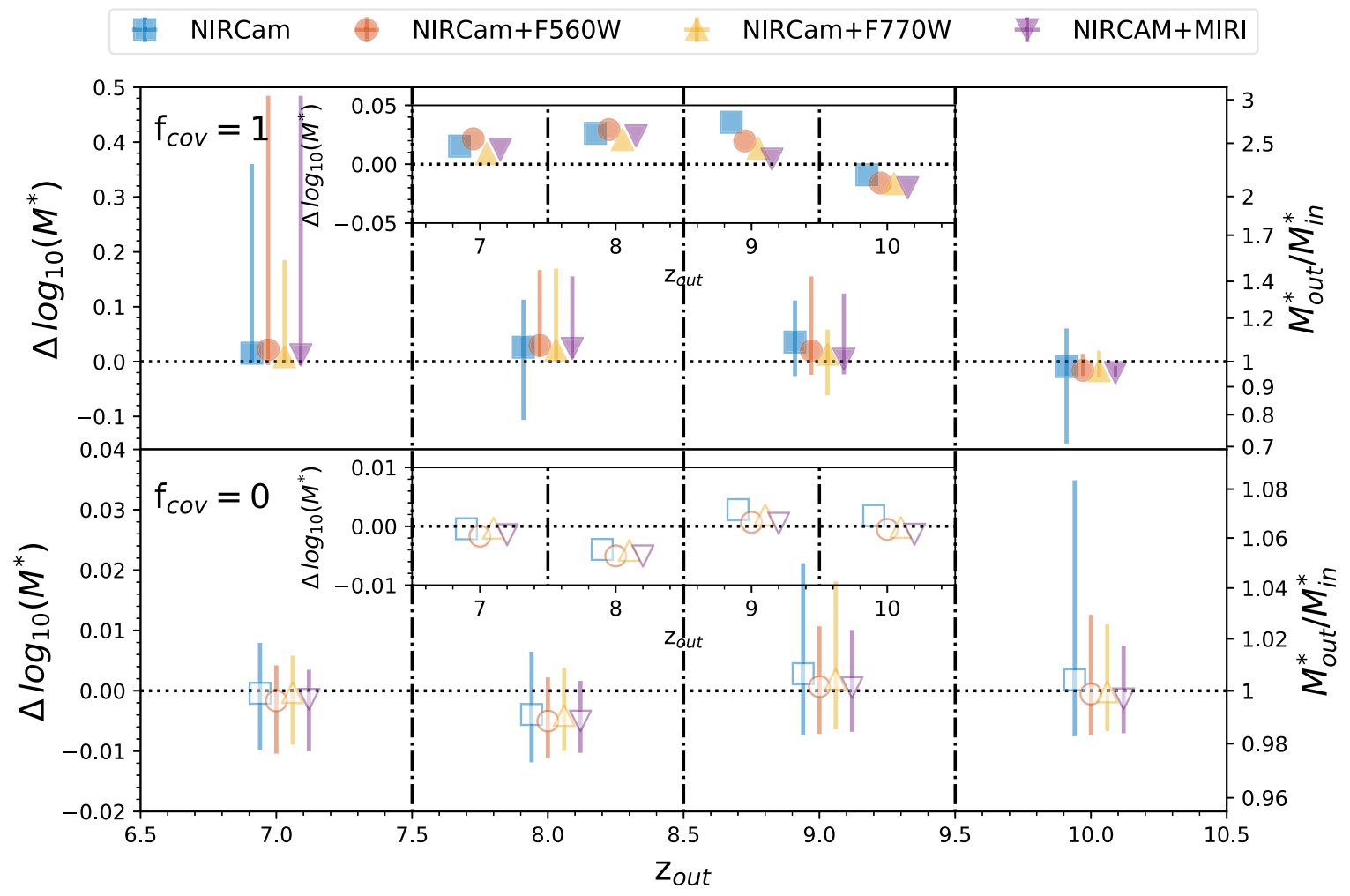

Figure 2. Median statistical stellar mass correction for different output redshift bins and broadband filter combinations: eight NIRCam broad bands (blue squares), eight NIRCam broad bands and MIRI F560W only (red circles), eight NIRCam broad bands and MIRI F770W only (yellow triangles), and eight NIRCam broad bands, MIRI F560W, and MIRI F770W (purple triangles). Galaxies are divided depending on their covering factor: $f_{\text {cov }}=1$ (top) and $f_{\text {cov }}=0$ (bottom). The vertical dotted-dashed lines indicate the edges of the considered redshift bins. Error bars correspond to the $25 \%$ and $75 \%$ quartiles. Points are slightly offset horizontally with respect to each other for illustrative purposes. The inset plots show a zoom-in on the median absolute values.

slightly changes to $3 \%$ when adding the MIRI broad bands. The stellar mass for the majority of these galaxies is overestimated independently by the considered filter combination, even up to 10 times in some cases, and more than $90 \%(60 \%)$ of them have stellar mass offsets larger than $0.1(0.3)$ dex in modulus. This is extremely high compared with the fraction of galaxies correctly identified as galaxies without emission lines, for which only $12 \%$ (2\%) have stellar mass errors larger than $0.1(0.3)$ dex in modulus, already considering only the eight NIRCam bands.

Overall, it is evident that the big offsets on the stellar mass arise from galaxies for which the covering factor, i.e., the presence of nebular emission lines, is wrongly recognized. However, even galaxies correctly identified as galaxies with nebular emission lines may also have large stellar mass statistical corrections. The inclusion of the MIRI bands generally reduces the fraction of galaxies wrongly identified as galaxies with nebular emission lines; therefore, it reduces the fraction of galaxies with extreme stellar mass offsets.

\subsection{Variation of Statistical Stellar Mass Correction with Redshift}

In Figure 2, we show the median statistical stellar mass correction for different redshift bins and filter combinations. We highlight once again that here we analyze output redshift values that do not necessarily correspond to the input ones, as will happen in real observations. All redshift bins are centered around the four input redshifts, $z=7,8,9$, and 10. We separately analyze galaxies with different covering factors, i.e., $f_{\text {cov }}=0$ or 1 .
Galaxies with output $f_{\text {cov }}=1$ are recognized as star-forming galaxies with the maximum contribution from nebular emission lines. They have generally small median stellar mass offsets between 0.036 and -0.020 dex, with negative values present at $z=10$. However, the distribution of the stellar mass correction is very broad in some cases, in particular at $z=7$, where the 75 th percentile is between 0.18 and 0.48 mag, depending on the considered filter. At $z=7$, extreme stellar mass offset is due to a overestimation of the age for the youngest galaxy template. Excluding $z=7$ templates, the addition of at least one MIRI band reduces the median, which is, however, already small using only the eight NIRCam broad bands, or the dispersion of the stellar mass offset, especially at $z=10$.

Galaxies with output $f_{\text {cov }}=0$ are recognized as galaxies without emission lines that could be both star-forming and quenched galaxies. The median stellar mass offsets are small, ranging between 0.005 and 0.003 dex. The $25 \%$ and $75 \%$ quartiles are always within \pm 0.04 dex, i.e., stellar mass offsets below $10 \%$, with a light dependence with redshift. The larger distribution is present at $z=10$, considering only NIRCam bands, and this is due to the fact that no NIRCam bands purely cover the $\lambda>4000 \AA$ break.

As also mentioned in B17, stellar masses are, on average, difficult to estimate for galaxies with nebular emission lines at all redshifts. Their stellar masses tend to be overestimated due to an overestimation of the galaxy age. In addition, stellar masses are also challenging to estimate for galaxies without nebular emission lines at $z=10$ when considering only NIRCam bands. 


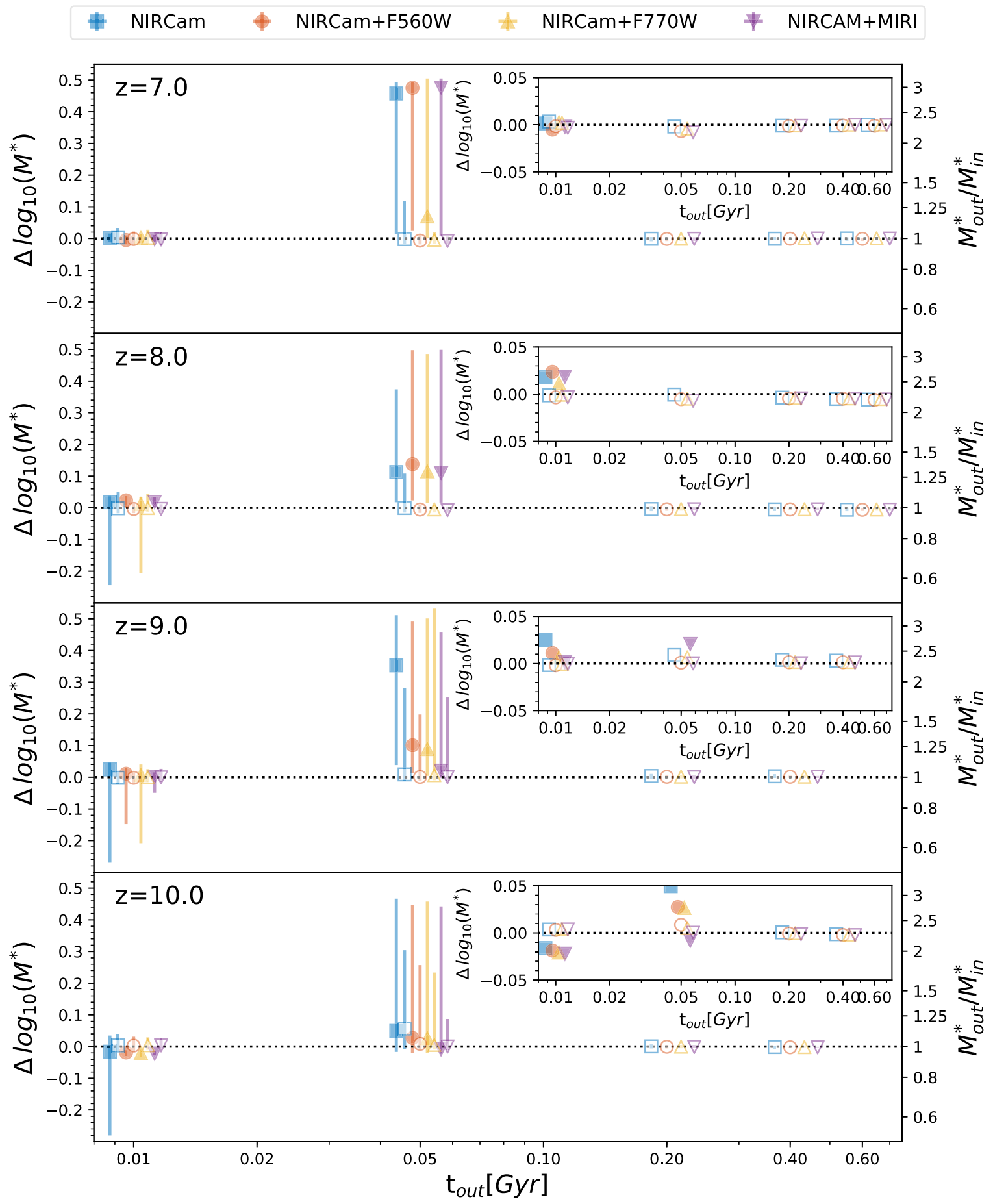

Figure 3. Median statistical stellar mass correction for different output ages and redshift bins. From top to bottom: redshifts $z=7,8,9$, and 10. Different symbols correspond to different broadband filter combinations: eight NIRCam broad bands (blue squares), eight NIRCam broad bands and MIRI F560W only (red circles), eight NIRCam broad bands and MIRI F770W only (yellow triangles), and eight NIRCam broad bands, MIRI F560W, and MIRI F770W (purple triangles). Filled symbols indicate galaxies with nebular emission lines, i.e., star-forming and with $f_{\text {cov }}=1$, while open symbols indicate galaxies without nebular emission lines, i.e., quiescent galaxies or star-forming galaxies with $f_{\text {cov }}=0$. Error bars correspond to the $25 \%$ and $75 \%$ quartiles. Points are slightly offset horizontally with respect to each other for illustrative purposes. The inset plots show a zoom-in on the median values.

\subsection{Variation of the Statistical Stellar Mass Correction with Galaxy Age}

Figure 3 shows the median statistical stellar mass correction for different output ages, redshifts, and filter combinations. Galaxies with $t_{\text {out }} \geqslant 0.20 \mathrm{Gyr}$ have stellar mass corrections within 0.01 dex, i.e., $\sim 2 \%$ error in the stellar mass estimation, including the $25 \%$ and $75 \%$ quartiles and considering all redshifts and JWST broadband filter combinations. These templates correspond to quiescent galaxies, for which stellar masses are generally correctly estimated. There are no starforming galaxies with $t_{\text {out }} \geqslant 0.20 \mathrm{Gyr}$, because stars can form only until $0.1 \mathrm{Gyr}$ in the galaxy models included in this work.

On the other hand, galaxies with ages equal to or smaller than $0.05 \mathrm{Gyr}$ are star-forming galaxies with or without 


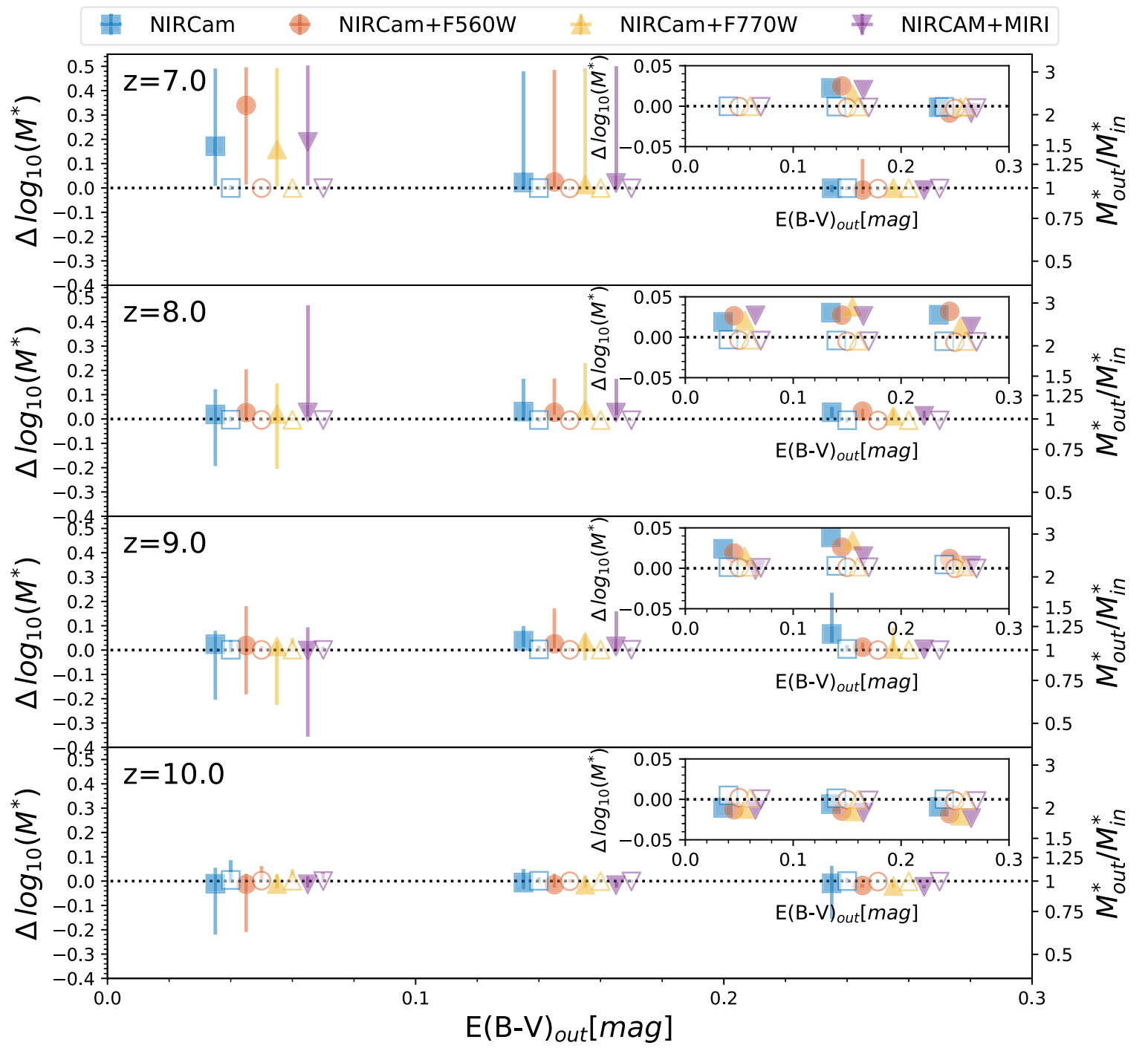

Figure 4. Median statistical stellar mass correction for different output color excess bins and redshift bins. From top to bottom: redshifts $z=7,8,9$, and 10 . Different symbols correspond to different broadband filter combinations: eight NIRCam broad bands (blue squares), eight NIRCam broad bands and MIRI F560W only (red circles), eight NIRCam broad bands and MIRI F770W only (yellow triangles), and eight NIRCam broad bands, MIRI F560W, and MIRI F770W (purple triangles). Filled symbols indicate galaxies with nebular emission lines, i.e., star-forming and with $f_{\text {cov }}=1$, while open symbols indicate galaxies without nebular emission lines, i.e., quiescent galaxies or star-forming galaxies with $f_{\text {cov }}=0$. Error bars correspond to the $25 \%$ and $75 \%$ quartiles. Points are slightly offset horizontally with respect to each other for illustrative purposes. The inset plots show a zoom-in on the median values.

emission lines, depending on the covering factors, or quiescent galaxies if the template corresponds to a very short period of star formation. In particular, galaxies with nebular emission lines, i.e., star-forming galaxies with $f_{\text {cov }}=1$, have stellar masses that are overestimated at $t=0.05 \mathrm{Gyr}$, up to $0.5 \mathrm{dex}$, and generally underestimated at $t=0.01 \mathrm{Gyr}$. At $z=7$, a large difference is present in the median offset values derived considering the MIRI/F770W band and both the F560W and F770W MIRI bands for galaxies with emission lines and $t=0.05$ Gyr. However, the distribution of the statistical stellar mass corrections of these galaxies is bimodal; therefore, a small difference in the overall distribution is enough to shift the median of $\sim 0.4$ dex.

Galaxies with $f_{\text {cov }}=0$ instead have a good stellar mass estimation for $t=0.01 \mathrm{Gyr}$ but may have overestimated stellar masses for $t=0.05 \mathrm{Gyr}$ at $z>8$. However, for a small fraction of these galaxies that have output $f_{\text {cov }}=0$ but nebular emission lines in input, the stellar mass is highly overestimated, even up to 1 dex. This generally results from an underestimation of the duration of star formation and an overestimation of the age.

\subsection{Variation of the Statistical Stellar Mass Correction with Color Excess}

In Figure 4, we show the median statistical stellar mass correction for different output color excess bins, output redshifts, and filter combinations. We differentiate again between galaxies with and without nebular emission lines. Galaxies without emission lines that could be quiescent galaxies or templates corresponding to star-forming systems with $f_{\text {cov }}=0$ have stellar mass correction quartiles within 0.05 dex, i.e., $\sim 12 \%$ error on the input stellar mass, for all output values of color excess and redshift. Stellar mass offsets are slightly larger, with quartiles that are within 0.1 dex, for galaxies without nebular emission lines at $z=10$ and $E(B-V) \sim 0.05$ mag when not including observations with the F770W MIRI band. At this high redshift, the F770W MIRI 


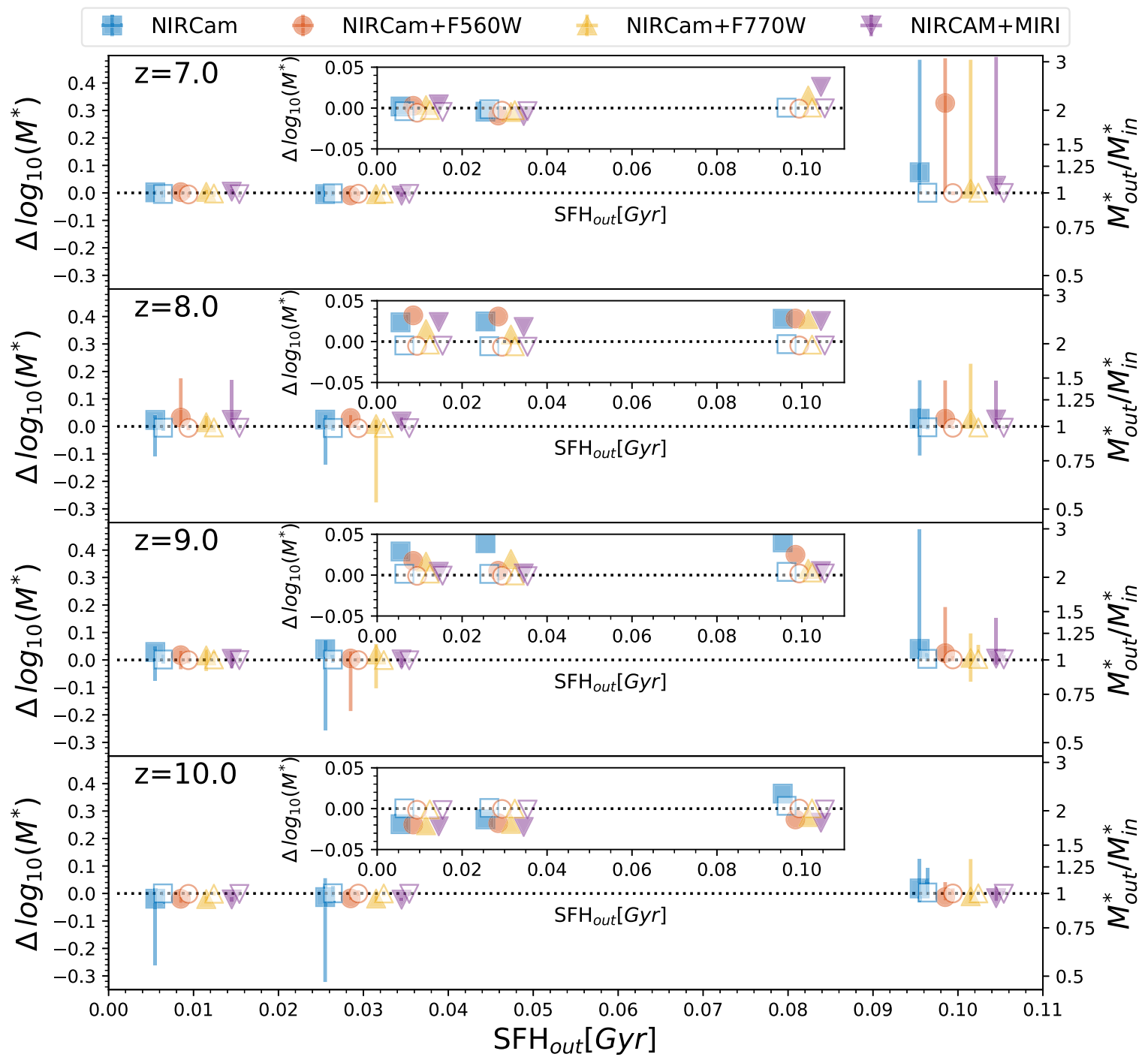

Figure 5. Median statistical stellar mass correction for different output star formation histories and redshift bins. From top to bottom: redshifts $z=7,8,9$, and 10 . Star formation histories are step functions that last for 0.01, 0.03, and 0.1 Gyr. Different symbols correspond to different broadband filter combinations: eight NIRCam broad bands (blue squares), eight NIRCam broad bands and MIRI F560W only (red circles), eight NIRCam broad bands and MIRI F770W only (yellow triangles), and eight NIRCam broad bands, MIRI F560W, and MIRI F770W (purple triangles). Filled symbols indicate galaxies with nebular emission lines, i.e., star-forming and with $f_{\text {cov }}=1$, while open symbols indicate galaxies without nebular emission lines, i.e., quiescent galaxies or star-forming galaxies with $f_{\text {cov }}=0$. Error bars correspond to the $25 \%$ and $75 \%$ quartiles. Points are slightly offset horizontally with respect to each other for illustrative purposes. The inset plots show a zoom-in on the median values.

band helps to estimate the dust extinction and stellar mass; otherwise, the first one is, on average, underestimated, and the second one is overestimated.

On the other hand, for galaxies with nebular emission lines, the stellar mass has a larger offset with respect to the input stellar mass than for galaxies without emission lines. In particular, the dispersion of the stellar mass correction distribution increases with decreasing values of the output color excess. Moreover, the stellar mass is generally overestimated at $z=7$, particularly for galaxies with $E(B-V)<0.1 \mathrm{mag}$, for which the median statistical stellar mass correction is between 0.16 and 0.34 dex. Conversely, at $z>7$, the stellar mass is underestimated for a nonnegligible fraction of galaxies with nebular emission lines, with 25th percentiles that also reach -0.36 dex; i.e., the stellar mass is underestimated by $56 \%$. At $z>8$, the stellar mass correction also has a large distribution for galaxies with $\langle E(B-V)\rangle \sim 0.25 \mathrm{mag}$, if only eight NIRCam bands are considered. Galaxies with $E(B-V)<0.1 \mathrm{mag}$, for which the stellar mass is underestimated, correspond to galaxies with input $f_{\mathrm{cov}}=1(\sim 25 \%)$, for which the age is slightly underestimated, as well as the period of star formation, or galaxies with input $f_{\text {cov }}=0(\sim 75 \%)$, for which the color excess and metallicity have been underestimated while star formation continues for a longer period than in the input.

Overall, stellar masses are generally well recovered for galaxies with an output color excess around 0.2-0.3 mag, while it is less accurate for galaxies for which the best SED template is relatively dust-free. This is due to the age-extinction degeneracy for galaxies with output $f_{\text {cov }}=0$ and more complicated degeneracies, which also involve the duration of star formation and the metallicity, for galaxies with output $f_{\text {cov }}=1$.

\subsection{Variation of the Statistical Stellar Mass Correction with $\mathrm{SFH}$}

Figure 5 shows the stellar mass corrections for different output SFHs, redshifts, and broadband filter combinations. In 


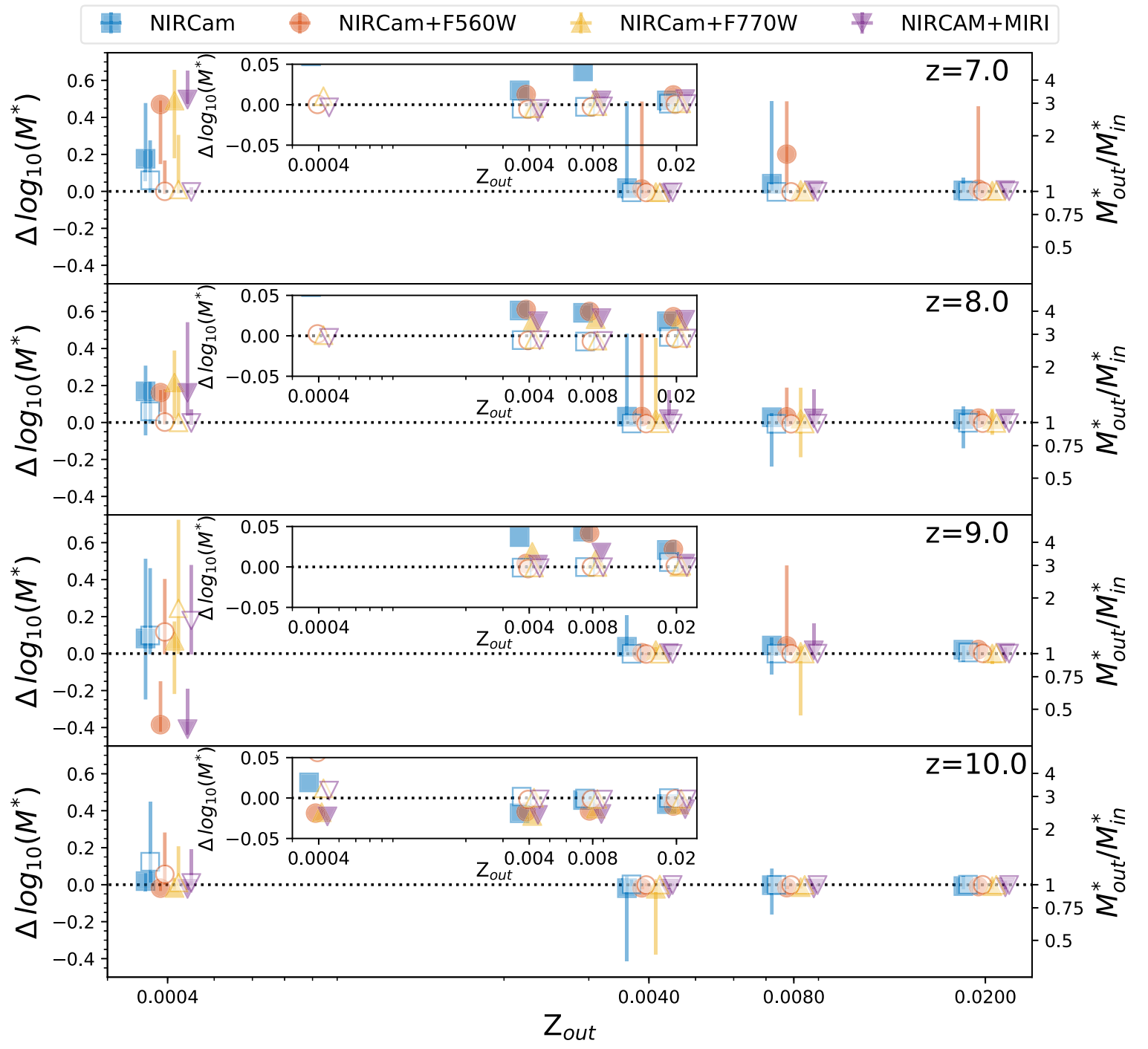

Figure 6. Median statistical stellar mass correction for different output metallicities and redshift bins. From top to bottom: redshifts $z=7,8,9$, and 10 . Different symbols correspond to different broadband filter combinations: eight NIRCam broad bands (blue squares), eight NIRCam broad bands and MIRI F560W only (red circles), eight NIRCam broad bands and MIRI F770W only (yellow triangles), and eight NIRCam broad bands, MIRI F560W, and MIRI F770W (purple triangles). Filled symbols indicate galaxies with nebular emission lines, i.e., star-forming and with $f_{\text {cov }}=1$, while open symbols indicate galaxies without nebular emission lines, i.e., quiescent galaxies or star-forming galaxies with $f_{\text {cov }}=0$. Error bars correspond to the $25 \%$ and $75 \%$ quartiles. Points are slightly offset horizontally with respect to each other for illustrative purposes. The inset plots show a zoom-in on the median values.

this work, we consider step function SFHs that last for 0.01, 0.03 , and $0.1 \mathrm{Gyr}$. Galaxies without emission lines have both quartiles of the stellar mass correction within 0.05 dex, excluding galaxies at $z=10$ and star formation lasting for $0.1 \mathrm{Gyr}$ observed using only the eight NIRCam bands, for which the $75 \%$ quartile of the stellar mass correction is 0.09 dex.

Galaxies with nebular emission lines have large stellar mass offsets, with $75 \%$ quartiles as large as $0.5 \mathrm{dex}$, particularly for galaxies with SFHs that last $0.1 \mathrm{Gyr}$ at $z<10$. Indeed, some of these galaxies are correctly identified as star-forming galaxies with nebular emission lines, but the SFH and age are overestimated, with a consequent underestimation of the line equivalent widths and, therefore, overestimation of the stellar mass. On the other hand, if the galaxy was originally without emission lines but wrongly identified with $f_{\text {cov }}=1$, the age has usually been largely underestimated, and, as a consequence, the stellar mass is overestimated because of a large mass-to-light ratio. If the SFH and age are underestimated, the line equivalent width is overestimated, and the stellar mass, consequently, is underestimated. This is the case for galaxies with SFHs shorter than $0.1 \mathrm{Gyr}$, for which the stellar mass tends to be underestimated down to -0.3 dex, i.e., around half of the input stellar mass, particularly when observed using only the eight NIRCam bands.

\subsection{Variation of the Stellar Mass Correction with Metallicity}

In Figure 6, we show the stellar mass correction for different output redshifts and metallicities and different broadband filter combinations. The largest scatter in the stellar mass correction is measured for galaxies with the lowest metallicity, i.e., $1 / 50 Z_{\odot}$. Galaxies without emission lines and with metallicities larger than $Z=0.0004$ have extremely small stellar mass corrections, with both quartiles within 0.025 dex. Instead, at the lowest considered 
metallicity, galaxies without emission lines may have stellar mass corrections with $75 \%$ quartiles as large as 0.72 dex.

On the other hand, galaxies with nebular emission lines have large stellar mass corrections at all considered metallicities. For galaxies with nebular emission lines, stellar masses may be largely overestimated, with $75 \%$ quartiles as large as 0.66 dex, as well as largely underestimated, with $25 \%$ quartiles down to -0.44 dex. When the stellar mass is overestimated, both the color excess and redshift are generally underestimated, while the age is overestimated. For galaxies with nebular emission lines, the SFH is generally underestimated and, because the age is overestimated, the equivalent widths of the nebular emission lines are underestimated. Because the equivalent widths are underestimated, the stellar mass is overestimated. As expected, when the stellar mass is underestimated, the opposite situation happens. For clarification, the redshift offsets are generally $\delta z=$ $\left|z_{\text {in }}-z_{\text {out }}\right| /\left(1+z_{\text {in }}\right)<0.15$, as there are almost no redshift outliers in the analyzed sample.

\section{How to Apply Corrections to Stellar Masses Based on JWST Observations}

In the previous sections, we have discussed the statistical stellar mass corrections derived from our mock galaxy catalog by analyzing for which templates the stellar mass estimation is particularly challenging. In this section, we give some practice information on how to include these results on the stellar mass estimation of galaxies observed with JWST in the near future.

First, we remind the reader that, under the assumption that emission lines depend on SFH, metallicity, covering factor, age, and dust extinction, but not on stellar mass, the stellar mass correction can be applied to any stellar mass and does not depend on the assumed stellar mass distribution. On the other hand, we advise using these corrections directly only for stellar masses derived using the LePhare code and the SED template considered in this work. If other codes or templates are used or small and biased samples are considered, like samples containing only emission-line galaxies, the stellar mass corrections can be used anyway as an indication of the possible degeneracies.

We provide the $25 \%, 50 \%$, and $75 \%$ quartiles and minimum and maximum values of the statistical stellar mass corrections for each combination of output galaxy parameters for galaxies with $f_{\text {cov }}=0$ (Table 2) and $f_{\text {cov }}=1$ (Table 3) separately. We also give the entire stellar mass offset distribution for each specific SED template for both $\mathrm{S} / \mathrm{N}=10$ and 20 and for the four JWST broadband filter combinations considered: eight NIRCam broad bands (Table 4), eight NIRCam broad bands and MIRI/F560W (Table 5), eight NIRCam broad bands and MIRI/F770W (Table 6), and eight NIRCam broad bands, MIRI/F560W, and MIRI/F770W (Table 7).

In general, given a sample of galaxies observed using a specific set of JWST filters, we suggest deriving the best SED template for each observed galaxy using the template utilized in this paper. After obtaining the best SED template, it is possible to consult Tables 2 or 3 , depending on the output covering factor, to derive the $25 \%, 50 \%$, and $75 \%$ quartiles of the stellar mass offset, or to look at Tables 4-7, depending on the available JWST bands, to obtain the full stellar mass offset distribution. We highlight that the stellar mass offsets presented in this work need to be considered as statistical corrections; therefore, their application to small samples or single objects is not recommended. They can be used on small or biased samples only to identify relatively secure or challenging galaxy templates for which the stellar mass estimation may be considered relatively secure or needs a further, more focused investigation.

It is possible to interpolate among stellar mass offset distributions with similar output parameter values; however, we suggest performing this interpolation with caution, particularly for galaxies with emission lines. Figures 7 and 8 show some examples of linear interpolation between available parameter values. In particular, in Figure 7, we show the interpolation between two templates with $z=7$ and 9, with all other parameters identical, and we compare the result with the corresponding template at $z=8$. In Figure 8, we repeat the exercise by changing the color excess and, in particular, by comparing the interpolation between a template with $\langle E(B-V)\rangle=0.05$ and 0.25 mag with the corresponding template with $\langle E(B-V)\rangle=0.15$ mag. The interpolated template never coincides perfectly with the real one, but it may resemble it, particularly for galaxies without emission lines. Therefore, we suggest interpolating with caution among the parameter values considered in this work.

\section{Comparison with the JAGUAR Catalog}

We test our method by applying the statistical stellar mass correction to the NIRCam mock observations derived as part of the JWST Advanced Deep Extragalactic Survey (JADES) using the JAdes extraGalactic Ultradeep Artificial Realizations (JAGUAR) package (Williams et al. 2018). These mock catalogs are derived by generating a galaxy population that follows empirical functions, such as the observed stellar mass and luminosity functions at different redshifts, and assigning each of them a SED template using the BayEsian Analysis of GaLaxy sEds (BEAGLE) tool (Chevallard \& Charlot 2016). We consider only mock galaxies between $z=6.5$ and 10.5 , for a total of 23,493 objects. We consider an observational depth of $10 \sigma$ at $29 \mathrm{AB}$ mag at $1.5 \mu \mathrm{m}$, and we consider only galaxies with $\mathrm{S} / \mathrm{N}_{\mathrm{F} 150 \mathrm{w}} \geqslant 10$.

We run the code LePhare using the same templates described in Section 2.3. We then correct the stellar mass function in the different redshift bins by considering the full stellar mass offset distribution associated with the best template obtained for each object (Table 4). In Figure 9, we compare the expected stellar mass corrections and the difference between the original and recovered stellar mass of the JAGUAR catalog before applying any correction. The overall magnitudes of the corrections are similar to the stellar mass discrepancies, and, even with a large scatter, they show a positive correlation, showing that the stellar mass correction generally improves the stellar mass estimation obtained with LePhare and the templates considered in this work. In line with the detailed analysis of the main degeneracies done in Section 3, we notice that solar metallicity systems tend to have a correct stellar mass estimation, while the stellar mass estimation is more challenging for objects with high star formation rates that present emission lines and objects with $t_{\text {out }}<0.2$ Gyr.

Figure 10 shows the difference between the stellar mass distribution of the JAGUAR catalog, the one derived using LePhare, and the Yggdrasil templates considered in this work 
Table 2

Statistical Stellar Mass Corrections $\Delta\left(\log _{10}\left(M^{*}\right)\right)=\log _{10}\left(M_{\text {out }}^{*} / M_{\odot}\right)-\log _{10}\left(M_{\text {in }}^{*} / M_{\odot}\right)$ for Different Output Galaxy Property Combinations for Galaxies with Output $f_{\text {cov }}=0$

\begin{tabular}{|c|c|c|c|c|c|c|c|c|c|c|c|c|c|c|c|c|c|c|c|c|c|c|c|c|}
\hline \multirow{3}{*}{$\begin{array}{l}\langle z\rangle \\
1 \\
1\end{array}$} & \multirow{3}{*}{$\begin{array}{c}Z \\
\left(Z_{\odot}\right) \\
2\end{array}$} & \multirow{3}{*}{$\begin{array}{c}\text { SFH } \\
(\mathrm{Gyr}) \\
3\end{array}$} & \multirow{3}{*}{$\begin{array}{c}t \\
(\mathrm{Gyr}) \\
4\end{array}$} & \multirow{3}{*}{$\begin{array}{c}\langle E(B-V)\rangle \\
(\mathrm{mag}) \\
5\end{array}$} & \multicolumn{5}{|c|}{ NIRCam } & \multirow{2}{*}{\multicolumn{5}{|c|}{ NIRCam }} & \multirow{2}{*}{\multicolumn{5}{|c|}{$\begin{array}{l}\text { NIRCam } \\
+ \text { F770W }\end{array}$}} & \multirow{2}{*}{\multicolumn{5}{|c|}{$\frac{\text { NIRCam }}{+ \text { F560W+F770W }}$}} \\
\hline & & & & & \multirow[b]{2}{*}{$25 \%$} & \multirow[b]{2}{*}{$50 \%$} & \multirow[b]{2}{*}{$\begin{array}{c}75 \% \\
6 \\
\end{array}$} & & & & & & & & & & & & & & & & & \\
\hline & & & & & & & & Min. & Max. & $25 \%$ & $50 \%$ & $\begin{array}{l}75 \% \\
7\end{array}$ & Min. & Max. & $25 \%$ & $50 \%$ & $\begin{array}{c}75 \% \\
8\end{array}$ & Min. & Max. & $25 \%$ & $50 \%$ & $\begin{array}{c}75 \% \\
9\end{array}$ & Min. & Max. \\
\hline 7 & 0.02 & 0.1 & 0.05 & 0.05 & 0.32 & 0.19 & 0.72 & -0.03 & 0.78 & 0.20 & 0.00 & 0.34 & -0.03 & 0.73 & 0.44 & 0.20 & 0.72 & -0.03 & 0.78 & 0.20 & 0.00 & 0.34 & -0.03 & 0.73 \\
\hline 7 & 0.02 & 0.01 & 0.01 & 0.15 & 0.16 & -0.01 & 0.30 & -0.27 & 0.48 & 0.00 & -0.02 & 0.20 & -0.24 & 0.32 & 0.02 & -0.01 & 0.31 & -0.22 & 0.48 & 0.00 & -0.02 & 0.03 & -0.23 & 0.33 \\
\hline 7 & 0.02 & 0.03 & 0.01 & 0.15 & 0.21 & -0.01 & 0.31 & -0.07 & 0.47 & 0.18 & -0.01 & 0.21 & -0.03 & 0.31 & 0.19 & 0.00 & 0.46 & -0.03 & 0.61 & 0.00 & -0.02 & 0.19 & -0.03 & 0.31 \\
\hline 7 & 0.02 & 0.1 & 0.01 & 0.15 & 0.19 & 0.01 & 0.28 & -0.06 & 0.49 & 0.03 & -0.01 & 0.23 & -0.05 & 0.32 & 0.18 & 0.00 & 0.31 & -0.03 & 0.61 & 0.00 & -0.02 & 0.19 & -0.03 & 0.32 \\
\hline 7 & 0.02 & 0.1 & 0.05 & 0.15 & 0.40 & 0.20 & 0.70 & -0.08 & 0.88 & 0.01 & -0.02 & 0.19 & -0.08 & 0.89 & 0.45 & 0.21 & 0.70 & -0.08 & 0.88 & 0.00 & -0.02 & 0.08 & -0.08 & 0.89 \\
\hline 7 & 0.02 & 0.03 & 0.01 & 0.25 & 0.12 & 0.00 & 0.23 & -0.05 & 0.51 & 0.00 & -0.01 & 0.03 & -0.05 & 0.33 & 0.00 & -0.03 & 0.02 & -0.13 & 0.59 & 0.00 & -0.02 & 0.01 & -0.12 & 0.24 \\
\hline 7 & 0.02 & 0.1 & 0.05 & 0.25 & 0.19 & 0.00 & 0.35 & -0.04 & 0.86 & 0.00 & -0.02 & 0.04 & -0.03 & 0.42 & 0.01 & -0.01 & 0.37 & -0.04 & 0.86 & -0.01 & -0.02 & 0.01 & -0.04 & 0.87 \\
\hline 8 & 0.02 & 0.1 & 0.05 & 0.05 & 0.27 & 0.09 & 0.45 & -0.02 & 0.74 & 0.29 & 0.01 & 0.36 & -0.02 & 0.58 & 0.31 & 0.01 & 0.46 & -0.02 & 0.74 & 0.29 & 0.00 & 0.36 & -0.02 & 0.58 \\
\hline 8 & 0.02 & 0.01 & 0.01 & 0.15 & 0.18 & -0.01 & 0.46 & -0.27 & 0.60 & 0.18 & -0.01 & 0.20 & -0.27 & 0.33 & 0.20 & 0.00 & 0.46 & -0.28 & 0.48 & 0.01 & -0.01 & 0.20 & -0.28 & 0.30 \\
\hline 8 & 0.02 & 0.03 & 0.01 & 0.15 & 0.20 & 0.04 & 0.39 & -0.10 & 0.59 & 0.20 & 0.18 & 0.22 & -0.27 & 0.33 & 0.23 & 0.18 & 0.46 & -0.27 & 0.59 & 0.20 & 0.19 & 0.21 & -0.03 & 0.32 \\
\hline 8 & 0.02 & 0.1 & 0.01 & 0.15 & 0.21 & 0.00 & 0.33 & -0.07 & 0.49 & 0.20 & -0.01 & 0.22 & -0.11 & 0.33 & 0.20 & 0.13 & 0.46 & -0.02 & 0.49 & 0.18 & -0.01 & 0.20 & -0.11 & 0.34 \\
\hline 8 & 0.02 & 0.03 & 0.05 & 0.15 & 0.10 & 0.00 & 0.12 & -0.04 & 0.35 & 0.00 & -0.01 & 0.12 & -0.11 & 0.39 & -0.01 & -0.02 & 0.00 & -0.11 & 0.46 & -0.01 & -0.02 & 0.00 & -0.10 & 0.39 \\
\hline 8 & 0.02 & 0.1 & 0.05 & 0.15 & 0.24 & 0.00 & 0.37 & -0.08 & 0.86 & 0.00 & -0.01 & 0.17 & -0.08 & 0.54 & 0.17 & 0.00 & 0.38 & -0.08 & 0.86 & 0.00 & -0.01 & 0.16 & -0.08 & 0.88 \\
\hline 8 & 0.02 & 0.1 & 0.05 & 0.25 & 0.17 & 0.01 & 0.33 & -0.04 & 0.75 & 0.10 & -0.01 & 0.32 & -0.05 & 0.48 & 0.00 & -0.01 & 0.02 & -0.06 & 0.39 & 0.00 & -0.01 & 0.01 & -0.06 & 0.42 \\
\hline 8 & 1 & 0.1 & 0.05 & 0.05 & 0.24 & 0.02 & 0.34 & -0.22 & 0.54 & 0.09 & 0.01 & 0.26 & -0.18 & 0.52 & 0.05 & 0.00 & 0.23 & -0.04 & 0.54 & 0.06 & 0.00 & 0.15 & -0.04 & 0.52 \\
\hline 9 & 0.02 & 0.01 & 0.05 & 0.05 & 0.23 & 0.01 & 0.88 & -0.07 & 0.90 & 0.10 & 0.00 & 0.41 & -0.07 & 0.91 & 0.87 & 0.00 & 0.89 & -0.07 & 0.92 & 0.01 & -0.01 & 0.87 & -0.06 & 0.91 \\
\hline 9 & 0.02 & 0.03 & 0.05 & 0.05 & 0.10 & -0.01 & 0.79 & -0.19 & 0.98 & 0.06 & -0.01 & 0.46 & -0.12 & 0.97 & 0.48 & 0.00 & 0.94 & -0.15 & 0.97 & 0.01 & -0.01 & 0.76 & -0.20 & 0.96 \\
\hline 9 & 0.02 & 0.1 & 0.05 & 0.05 & 0.29 & 0.02 & 0.70 & -0.06 & 0.76 & 0.30 & 0.22 & 0.72 & -0.06 & 0.76 & 0.46 & 0.24 & 0.73 & -0.06 & 0.76 & 0.47 & 0.25 & 0.73 & -0.16 & 0.76 \\
\hline 9 & 0.02 & 0.01 & 0.01 & 0.15 & 0.01 & -0.01 & 0.42 & -0.27 & 0.49 & 0.13 & -0.01 & 0.44 & -0.30 & 0.65 & 0.20 & 0.00 & 0.46 & -0.42 & 0.65 & 0.20 & 0.00 & 0.47 & -0.30 & 0.65 \\
\hline 9 & 0.02 & 0.03 & 0.01 & 0.15 & 0.01 & -0.01 & 0.28 & -0.13 & 0.50 & 0.11 & -0.01 & 0.25 & -0.26 & 0.50 & 0.19 & 0.00 & 0.21 & -0.27 & 0.61 & 0.20 & 0.00 & 0.27 & -0.26 & 0.63 \\
\hline 9 & 0.02 & 0.1 & 0.01 & 0.15 & 0.01 & -0.01 & 0.24 & -0.27 & 0.49 & 0.13 & 0.00 & 0.22 & -0.27 & 0.64 & 0.22 & 0.07 & 0.48 & -0.31 & 0.65 & 0.21 & 0.01 & 0.61 & -0.26 & 0.65 \\
\hline 9 & 0.02 & 0.1 & 0.05 & 0.15 & 0.39 & 0.17 & 0.72 & -0.12 & 0.90 & 0.41 & 0.24 & 0.73 & -0.14 & 0.91 & 0.72 & 0.26 & 0.74 & -0.26 & 0.91 & 0.71 & 0.25 & 0.87 & -0.14 & 0.92 \\
\hline 9 & 0.02 & 0.01 & 0.01 & 0.25 & 0.00 & -0.01 & 0.28 & -0.31 & 0.49 & 0.21 & 0.00 & 0.47 & -0.27 & 0.65 & 0.00 & -0.01 & 0.20 & -0.23 & 0.62 & 0.46 & 0.00 & 0.48 & -0.22 & 0.65 \\
\hline 9 & 0.02 & 0.03 & 0.01 & 0.25 & 0.20 & 0.00 & 0.41 & -0.11 & 0.49 & 0.16 & 0.00 & 0.40 & -0.07 & 0.66 & 0.00 & -0.01 & 0.01 & -0.07 & 0.62 & 0.00 & -0.01 & 0.02 & -0.08 & 0.66 \\
\hline 9 & 0.02 & 0.01 & 0.05 & 0.25 & 0.39 & 0.00 & 0.87 & -0.09 & 0.90 & -0.01 & -0.01 & 0.01 & -0.07 & 0.40 & 0.86 & 0.00 & 0.87 & -0.04 & 0.89 & -0.01 & -0.01 & 0.00 & -0.03 & 0.21 \\
\hline 9 & 0.02 & 0.1 & 0.05 & 0.25 & 0.66 & 0.25 & 0.70 & -0.13 & 0.89 & 0.23 & 0.01 & 0.38 & -0.12 & 0.89 & 0.70 & 0.26 & 0.72 & -0.08 & 0.91 & 0.39 & 0.01 & 0.70 & -0.07 & 0.90 \\
\hline 9 & 0.2 & 0.1 & 0.05 & 0.05 & 0.19 & 0.00 & 0.34 & -0.33 & 0.73 & 0.01 & -0.01 & 0.28 & -0.32 & 0.71 & 0.19 & 0.00 & 0.49 & -0.33 & 0.73 & 0.00 & -0.01 & 0.28 & -0.32 & 0.71 \\
\hline 9 & 0.2 & 0.03 & 0.01 & 0.15 & 0.00 & -0.08 & 0.01 & -0.46 & 0.44 & 0.00 & -0.05 & 0.02 & -0.46 & 0.45 & 0.01 & -0.01 & 0.41 & -0.53 & 0.45 & 0.40 & -0.01 & 0.42 & -0.39 & 0.45 \\
\hline 9 & 0.4 & 0.1 & 0.05 & 0.05 & 0.22 & 0.00 & 0.48 & -0.30 & 0.70 & 0.06 & 0.00 & 0.29 & -0.32 & 0.68 & 0.19 & 0.00 & 0.48 & -0.33 & 0.69 & 0.08 & 0.00 & 0.39 & -0.32 & 0.57 \\
\hline 9 & 1 & 0.1 & 0.05 & 0.25 & 0.34 & 0.00 & 0.41 & -0.30 & 0.48 & 0.00 & -0.03 & 0.24 & -0.31 & 0.49 & -0.01 & -0.03 & 0.01 & -0.11 & 0.54 & 0.00 & -0.02 & 0.02 & -0.13 & 0.05 \\
\hline 10 & 0.02 & 0.03 & 0.05 & 0.05 & 0.11 & 0.01 & 0.80 & -0.13 & 0.83 & 0.09 & 0.00 & 0.35 & -0.13 & 0.98 & 0.00 & -0.01 & 0.05 & -0.13 & 0.98 & 0.00 & -0.01 & 0.07 & -0.12 & 0.98 \\
\hline 10 & 0.02 & 0.1 & 0.05 & 0.05 & 0.34 & 0.25 & 0.56 & -0.03 & 0.77 & 0.32 & 0.22 & 0.48 & -0.16 & 0.75 & 0.32 & 0.26 & 0.47 & -0.03 & 0.76 & 0.32 & 0.22 & 0.47 & -0.03 & 0.76 \\
\hline 10 & 0.02 & 0.01 & 0.01 & 0.15 & 0.02 & -0.02 & 0.28 & -0.28 & 0.50 & 0.07 & 0.02 & 0.28 & -0.28 & 0.48 & 0.21 & 0.04 & 0.29 & -0.29 & 0.30 & 0.21 & 0.02 & 0.29 & -0.28 & 0.30 \\
\hline 10 & 0.02 & 0.03 & 0.01 & 0.15 & 0.29 & 0.02 & 0.30 & -0.02 & 0.48 & 0.18 & 0.01 & 0.29 & -0.27 & 0.49 & 0.28 & 0.22 & 0.29 & -0.28 & 0.30 & 0.28 & 0.22 & 0.29 & -0.27 & 0.30 \\
\hline 10 & 0.02 & 0.1 & 0.01 & 0.15 & 0.29 & 0.07 & 0.46 & -0.02 & 0.49 & 0.19 & -0.02 & 0.21 & -0.08 & 0.46 & 0.21 & 0.20 & 0.28 & -0.08 & 0.44 & 0.20 & -0.01 & 0.23 & -0.08 & 0.44 \\
\hline 10 & 0.02 & 0.1 & 0.05 & 0.15 & 0.51 & 0.17 & 0.71 & -0.09 & 0.89 & 0.22 & 0.01 & 0.54 & -0.07 & 0.76 & 0.20 & 0.01 & 0.48 & -0.11 & 0.57 & 0.13 & 0.01 & 0.46 & -0.10 & 0.57 \\
\hline 10 & 0.02 & 0.03 & 0.01 & 0.25 & 0.28 & 0.20 & 0.30 & -0.08 & 0.48 & 0.20 & 0.00 & 0.22 & -0.05 & 0.30 & 0.00 & 0.00 & 0.01 & -0.03 & 0.45 & 0.00 & 0.00 & 0.01 & -0.03 & 0.43 \\
\hline 10 & 0.02 & 0.1 & 0.01 & 0.25 & 0.29 & 0.10 & 0.30 & -0.29 & 0.45 & 0.20 & 0.01 & 0.29 & -0.06 & 0.31 & 0.00 & -0.01 & 0.02 & -0.05 & 0.42 & 0.00 & -0.01 & 0.01 & -0.04 & 0.32 \\
\hline 10 & 0.02 & 0.03 & 0.05 & 0.25 & 0.12 & 0.00 & 0.82 & -0.03 & 0.84 & 0.00 & -0.01 & 0.01 & -0.04 & 0.83 & -0.01 & -0.01 & 0.00 & -0.04 & 0.38 & -0.01 & -0.01 & 0.00 & -0.04 & 0.15 \\
\hline 10 & 0.02 & 0.1 & 0.05 & 0.25 & 0.30 & 0.20 & 0.68 & -0.06 & 0.87 & 0.27 & 0.01 & 0.33 & -0.11 & 0.76 & 0.01 & 0.00 & 0.02 & -0.11 & 0.57 & 0.00 & -0.01 & 0.01 & -0.10 & 0.56 \\
\hline 10 & 0.2 & 0.1 & 0.05 & 0.05 & 0.28 & 0.08 & 0.52 & -0.20 & 0.73 & 0.29 & 0.03 & 0.56 & -0.16 & 0.73 & 0.28 & 0.02 & 0.58 & -0.18 & 0.73 & 0.25 & 0.01 & 0.57 & -0.20 & 0.73 \\
\hline 10 & 0.2 & 0.1 & 0.05 & 0.15 & 0.20 & 0.00 & 0.69 & -0.25 & 0.72 & 0.02 & -0.01 & 0.23 & -0.24 & 0.72 & 0.22 & 0.00 & 0.70 & -0.24 & 0.72 & 0.01 & -0.01 & 0.24 & -0.24 & 0.72 \\
\hline 10 & 0.2 & 0.1 & 0.05 & 0.25 & 0.28 & 0.02 & 0.68 & -0.24 & 0.72 & 0.01 & -0.01 & 0.22 & -0.23 & 0.72 & 0.69 & 0.00 & 0.71 & -0.10 & 0.73 & 0.00 & -0.01 & 0.02 & -0.09 & 0.73 \\
\hline 10 & 0.4 & 0.1 & 0.05 & 0.05 & 0.27 & 0.08 & 0.37 & -0.27 & 0.71 & 0.25 & 0.07 & 0.37 & -0.27 & 0.71 & 0.14 & 0.04 & 0.31 & -0.27 & 0.71 & 0.13 & 0.02 & 0.29 & -0.27 & 0.71 \\
\hline 10 & 1 & 0.1 & 0.05 & 0.05 & 0.12 & 0.05 & 0.34 & -0.23 & 0.54 & 0.12 & 0.05 & 0.33 & -0.22 & 0.55 & 0.12 & 0.05 & 0.34 & -0.23 & 0.55 & 0.11 & 0.05 & 0.27 & -0.23 & 0.55 \\
\hline 10 & 1 & 0.03 & 0.05 & 0.15 & 0.13 & 0.01 & 0.20 & -0.09 & 0.63 & 0.07 & 0.00 & 0.19 & -0.04 & 0.66 & 0.07 & 0.00 & 0.20 & -0.10 & 0.77 & 0.00 & -0.01 & 0.06 & -0.05 & 0.66 \\
\hline 10 & 1 & 0.1 & 0.05 & 0.25 & 0.25 & 0.00 & 0.35 & -0.25 & 0.53 & 0.01 & -0.01 & 0.27 & -0.24 & 0.54 & 0.00 & -0.01 & 0.01 & -0.06 & 0.36 & 0.00 & -0.02 & 0.01 & -0.06 & 0.33 \\
\hline
\end{tabular}

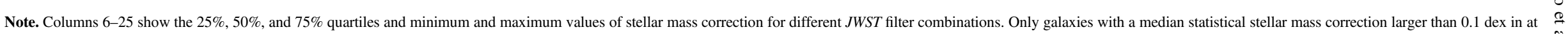
least one JWST filter combination are shown, but the complete table with all galaxy models is available online. A value of -99.9 is present when there are no galaxies for a combination of output galaxy properties.

(This table is available in its entirety in machine-readable form.) 
Table 3

Statistical Stellar Mass Corrections $\Delta\left(\log _{10}\left(M^{*}\right)\right)=\log _{10}\left(M_{\text {out }}^{*} / M_{\odot}\right)-\log _{10}\left(M_{\text {in }}^{*} / M_{\odot}\right)$ for Different Output Galaxy Property Combinations for Galaxies with Output $f_{\text {cov }}=1$

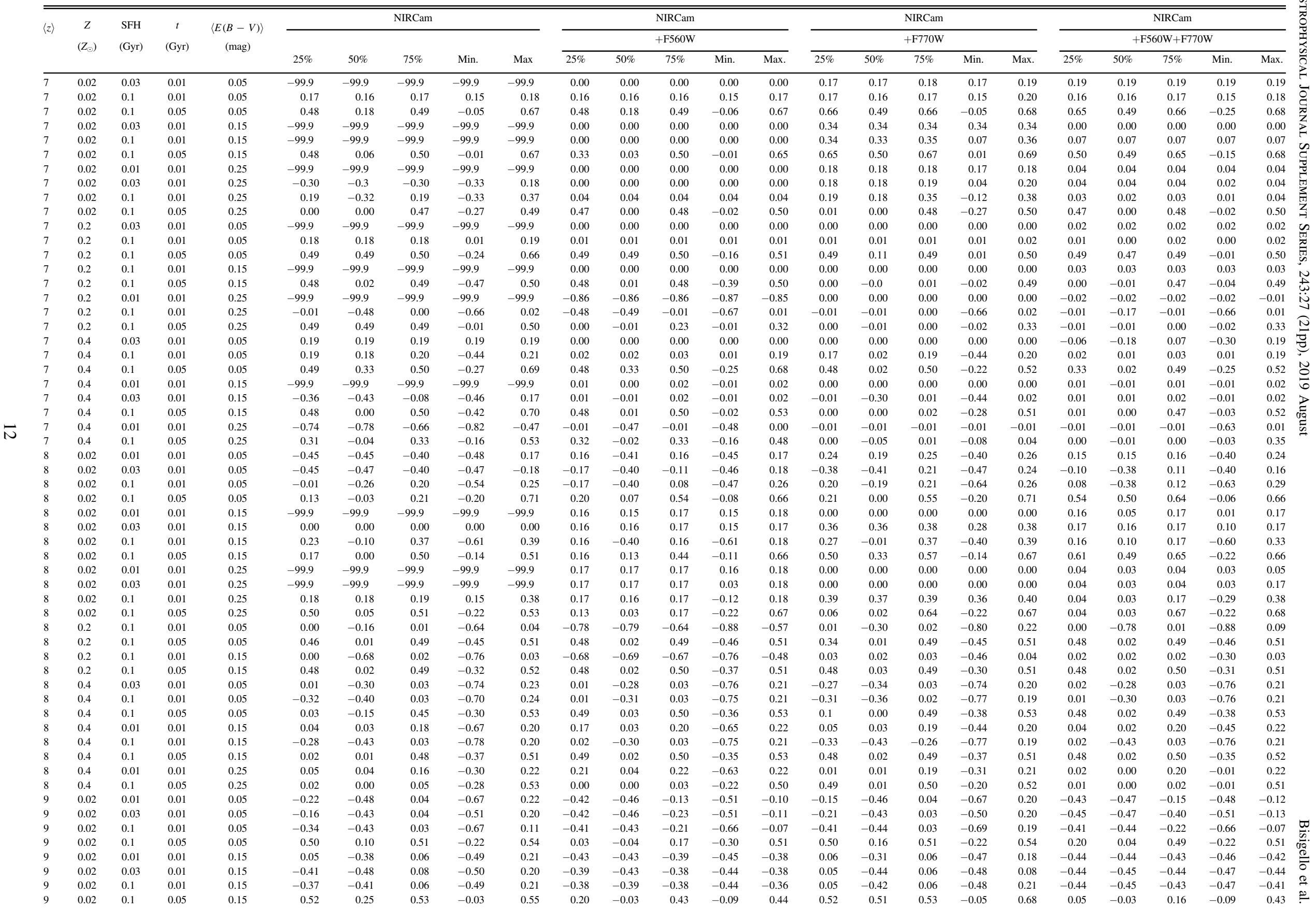


Table 3

(Continued)

\begin{tabular}{|c|c|c|c|c|c|c|c|c|c|c|c|c|c|c|c|c|c|c|c|c|c|c|c|c|}
\hline \multirow{3}{*}{$\langle z\rangle$} & \multirow{3}{*}{$\begin{array}{c}Z \\
\left(Z_{\odot}\right)\end{array}$} & \multirow{3}{*}{$\begin{array}{l}\text { SFH } \\
\text { (Gyr) }\end{array}$} & \multirow{3}{*}{$\begin{array}{c}{ }^{t} \\
(\mathrm{Gyr})\end{array}$} & \multirow{3}{*}{$\begin{array}{c}\langle E(B-V)\rangle \\
(\mathrm{mag})\end{array}$} & \multicolumn{5}{|c|}{ NIRCam } & \multicolumn{5}{|c|}{ NIRCam } & \multicolumn{5}{|c|}{ NIRCam } & \multicolumn{5}{|c|}{ NIRCam } \\
\hline & & & & & \multirow[b]{2}{*}{$25 \%$} & \multirow[b]{2}{*}{$50 \%$} & \multirow[b]{2}{*}{$75 \%$} & \multirow[b]{2}{*}{ Min. } & \multirow[b]{2}{*}{$\operatorname{Max}$} & \multicolumn{5}{|c|}{$+\mathrm{F} 560 \mathrm{~W}$} & \multicolumn{5}{|c|}{$+\mathrm{F} 770 \mathrm{~W}$} & \multicolumn{5}{|c|}{$+\mathrm{F} 560 \mathrm{~W}+\mathrm{F} 770 \mathrm{~W}$} \\
\hline & & & & & & & & & & $25 \%$ & $50 \%$ & $75 \%$ & Min. & Max. & $25 \%$ & $50 \%$ & $75 \%$ & Min. & Max. & $25 \%$ & $50 \%$ & $75 \%$ & Min. & Max. \\
\hline 9 & 0.02 & 0.01 & 0.01 & 0.25 & 0.09 & -0.07 & 0.10 & -0.42 & 0.11 & -0.40 & -0.4 & -0.40 & -0.41 & -0.17 & 0.09 & 0.08 & 0.10 & -0.42 & 0.25 & -0.40 & -0.41 & -0.40 & -0.41 & -0.39 \\
\hline 9 & 0.02 & 0.03 & 0.01 & 0.25 & 0.09 & -0.07 & 0.10 & -0.41 & 0.22 & -0.40 & -0.4 & -0.17 & -0.41 & -0.09 & 0.09 & 0.09 & 0.10 & -0.64 & 0.26 & -0.40 & -0.4 & -0.39 & -0.41 & -0.11 \\
\hline 9 & 0.02 & 0.1 & 0.01 & 0.25 & -99.9 & -99.9 & -99.9 & -99.9 & -99.9 & -0.16 & -0.17 & -0.09 & -0.62 & -0.07 & 0.09 & 0.07 & 0.10 & -0.59 & 0.25 & 0.00 & 0.00 & 0.00 & 0.00 & 0.00 \\
\hline 9 & 0.02 & 0.1 & 0.05 & 0.25 & 0.54 & 0.53 & 0.55 & -0.21 & 0.58 & 0.00 & -0.07 & 0.01 & -0.20 & 0.01 & 0.53 & 0.07 & 0.54 & 0.03 & 0.71 & 0.00 & 0.00 & 0.01 & -0.21 & 0.01 \\
\hline 9 & 0.2 & 0.01 & 0.01 & 0.05 & -99.9 & -99.9 & -99.9 & -99.9 & -99.9 & 0.00 & 0.00 & 0.00 & 0.00 & 0.00 & 0.03 & 0.01 & 0.03 & -0.69 & 0.06 & 0.00 & 0.00 & 0.00 & 0.00 & 0.00 \\
\hline 9 & 0.2 & 0.03 & 0.01 & 0.05 & -99.9 & -99.9 & -99.9 & -99.9 & -99.9 & 0.00 & 0.00 & 0.00 & 0.00 & 0.00 & 0.01 & 0.00 & 0.03 & -0.68 & 0.20 & 0.00 & 0.00 & 0.00 & 0.00 & 0.00 \\
\hline 9 & 0.2 & 0.1 & 0.01 & 0.05 & 0.01 & -0.44 & 0.03 & -0.85 & 0.20 & -0.83 & -0.83 & -0.66 & -0.84 & -0.48 & 0.01 & -0.39 & 0.03 & -0.87 & 0.06 & -0.48 & -0.83 & -0.44 & -0.84 & -0.29 \\
\hline 9 & 0.2 & 0.1 & 0.05 & 0.05 & 0.48 & 0.02 & 0.50 & -0.47 & 0.53 & -0.21 & -0.34 & 0.47 & -0.47 & 0.49 & 0.03 & 0.01 & 0.36 & -0.45 & 0.51 & 0.45 & -0.17 & 0.47 & -0.40 & 0.49 \\
\hline 9 & 0.2 & 0.01 & 0.01 & 0.15 & 0.04 & 0.03 & 0.05 & -0.47 & 0.22 & -0.17 & -0.33 & -0.01 & -0.33 & 0.00 & 0.04 & 0.04 & 0.05 & -0.47 & 0.07 & 0.00 & -0.09 & 0.00 & -0.32 & 0.00 \\
\hline 9 & 0.2 & 0.1 & 0.01 & 0.15 & 0.02 & -0.42 & 0.03 & -0.81 & 0.20 & -0.27 & -0.55 & 0.00 & -0.55 & 0.01 & 0.02 & -0.45 & 0.04 & -0.80 & 0.07 & 0.00 & -0.0 & 0.01 & -0.01 & 0.01 \\
\hline 9 & 0.2 & 0.1 & 0.05 & 0.15 & 0.49 & 0.03 & 0.51 & -0.25 & 0.54 & 0.47 & 0.46 & 0.48 & -0.19 & 0.49 & 0.01 & 0.00 & 0.05 & -0.02 & 0.54 & 0.46 & 0.00 & 0.47 & -0.28 & 0.48 \\
\hline 9 & 0.2 & 0.1 & 0.05 & 0.25 & 0.49 & 0.04 & 0.51 & -0.10 & 0.69 & 0.01 & 0.00 & 0.01 & -0.15 & 0.02 & 0.00 & -0.01 & 0.01 & -0.02 & 0.01 & 0.00 & -0.01 & 0.01 & -0.17 & 0.01 \\
\hline 9 & 0.4 & 0.01 & 0.01 & 0.05 & 0.04 & -0.28 & 0.05 & -0.69 & 0.23 & -0.36 & -0.37 & -0.03 & -0.42 & 0.18 & 0.03 & 0.01 & 0.04 & -0.52 & 0.07 & -0.03 & -0.37 & 0.01 & -0.51 & 0.18 \\
\hline 9 & 0.4 & 0.03 & 0.01 & 0.05 & 0.02 & -0.44 & 0.04 & -0.76 & 0.22 & 0.02 & -0.41 & 0.04 & -0.77 & 0.19 & -0.17 & -0.43 & 0.03 & -0.73 & 0.06 & 0.02 & -0.02 & 0.03 & -0.51 & 0.16 \\
\hline 9 & 0.4 & 0.1 & 0.01 & 0.05 & 0.02 & -0.38 & 0.04 & -0.84 & 0.22 & 0.02 & 0.01 & 0.03 & -0.75 & 0.20 & -0.37 & -0.40 & 0.03 & -0.74 & 0.06 & -0.04 & -0.38 & 0.01 & -0.75 & 0.18 \\
\hline 9 & 0.4 & 0.1 & 0.05 & 0.05 & 0.29 & 0.03 & 0.51 & -0.38 & 0.53 & 0.49 & 0.08 & 0.50 & -0.38 & 0.65 & 0.02 & -0.04 & 0.37 & -0.38 & 0.52 & 0.48 & 0.02 & 0.49 & -0.38 & 0.65 \\
\hline 9 & 0.4 & 0.01 & 0.01 & 0.15 & 0.05 & 0.04 & 0.06 & -0.50 & 0.23 & 0.16 & 0.00 & 0.17 & -0.46 & 0.19 & 0.05 & 0.04 & 0.05 & -0.46 & 0.07 & 0.00 & -0.01 & 0.16 & -0.46 & 0.17 \\
\hline 9 & 0.4 & 0.03 & 0.01 & 0.15 & 0.05 & -0.27 & 0.05 & -0.63 & 0.23 & 0.17 & 0.16 & 0.18 & -0.31 & 0.19 & 0.03 & -0.40 & 0.05 & -0.73 & 0.06 & 0.15 & 0.01 & 0.16 & -0.47 & 0.17 \\
\hline 9 & 0.4 & 0.1 & 0.01 & 0.15 & 0.04 & -0.35 & 0.06 & -0.55 & 0.24 & 0.17 & 0.01 & 0.18 & -0.74 & 0.19 & -0.41 & -0.42 & -0.33 & -0.56 & 0.17 & 0.16 & 0.00 & 0.16 & -0.73 & 0.17 \\
\hline 9 & 0.4 & 0.1 & 0.05 & 0.15 & 0.10 & 0.03 & 0.51 & -0.33 & 0.54 & 0.48 & 0.03 & 0.49 & -0.35 & 0.52 & 0.01 & 0.00 & 0.47 & -0.35 & 0.48 & 0.47 & 0.02 & 0.48 & -0.34 & 0.51 \\
\hline 9 & 0.4 & 0.01 & 0.01 & 0.25 & 0.07 & 0.06 & 0.17 & -0.31 & 0.21 & 0.01 & -0.29 & 0.16 & -0.46 & 0.17 & -0.15 & -0.31 & 0.04 & -0.47 & 0.24 & 0.01 & 0.00 & 0.15 & -0.47 & 0.16 \\
\hline 9 & 0.4 & 0.1 & 0.01 & 0.25 & 0.06 & -0.61 & 0.18 & -0.64 & 0.24 & 0.15 & 0.01 & 0.17 & 0.00 & 0.18 & 0.00 & 0.00 & 0.15 & -0.41 & 0.25 & 0.01 & 0.00 & 0.15 & -0.01 & 0.16 \\
\hline 9 & 0.4 & 0.1 & 0.05 & 0.25 & 0.37 & 0.07 & 0.50 & -0.16 & 0.68 & 0.01 & 0.01 & 0.01 & -0.13 & 0.48 & 0.01 & 0.00 & 0.03 & -0.01 & 0.47 & 0.00 & 0.00 & 0.01 & -0.15 & 0.46 \\
\hline 10 & 0.02 & 0.1 & 0.05 & $0 . c$ & 0.14 & 0.03 & 0.45 & -0.27 & 0.66 & 0.44 & 0.02 & 0.50 & -0.35 & 0.63 & 0.44 & 0.02 & 0.46 & -0.35 & 0.64 & 0.44 & 0.03 & 0.49 & -0.36 & 0.64 \\
\hline 10 & 0.02 & 0.1 & 0.05 & 0.15 & 0.30 & 0.04 & 0.45 & -0.28 & 0.64 & 0.12 & -0.01 & 0.45 & -0.34 & 0.47 & 0.12 & -0.02 & 0.45 & -0.27 & 0.64 & 0.10 & -0.02 & 0.45 & -0.27 & 0.61 \\
\hline 10 & 0.02 & 0.1 & 0.05 & 0.25 & 0.43 & 0.01 & 0.44 & -0.28 & 0.76 & -0.01 & -0.02 & 0.45 & -0.28 & 0.51 & -0.02 & -0.03 & 0.04 & -0.24 & 0.47 & -0.02 & -0.03 & 0.12 & -0.24 & 0.47 \\
\hline 10 & 0.2 & 0.1 & 0.01 & 0.05 & -0.07 & -0.47 & 0.01 & -0.87 & 0.04 & -0.02 & -0.51 & -0.01 & -0.95 & 0.01 & -0.13 & -0.46 & -0.01 & -0.93 & 0.03 & -0.02 & -0.03 & -0.01 & -0.51 & 0.00 \\
\hline 10 & 0.2 & 0.1 & 0.05 & 0.05 & 0.00 & -0.31 & 0.47 & -0.46 & 0.50 & 0.27 & -0.02 & 0.47 & -0.52 & 0.51 & -0.01 & -0.03 & 0.46 & -0.53 & 0.50 & -0.01 & -0.03 & 0.46 & -0.54 & 0.50 \\
\hline 10 & 0.2 & 0.01 & 0.01 & 0.15 & -0.16 & -0.42 & 0.04 & -0.81 & 0.21 & -0.01 & -0.02 & -0.01 & -0.02 & -0.01 & -0.02 & -0.03 & -0.01 & -0.73 & 0.03 & -0.02 & -0.02 & -0.01 & -0.49 & 0.00 \\
\hline 10 & 0.2 & 0.03 & 0.01 & 0.15 & -0.15 & -0.69 & -0.02 & -0.82 & 0.03 & -0.02 & -0.02 & -0.01 & -0.82 & 0.00 & -0.02 & -0.46 & -0.01 & -0.79 & 0.05 & -0.02 & -0.02 & -0.01 & -0.81 & -0.00 \\
\hline 10 & 0.2 & 0.1 & 0.01 & 0.15 & -0.01 & -0.49 & 0.03 & -0.82 & 0.05 & -0.03 & -0.70 & -0.02 & -0.82 & -0.01 & -0.45 & -0.50 & -0.02 & -0.82 & 0.06 & -0.49 & -0.51 & -0.02 & -0.82 & -0.01 \\
\hline 10 & 0.2 & 0.1 & 0.01 & 0.25 & -0.02 & -0.50 & 0.04 & -0.82 & 0.08 & -0.02 & -0.49 & -0.02 & -0.81 & -0.01 & -0.49 & -0.50 & -0.02 & -0.81 & -0.01 & -0.03 & -0.49 & -0.02 & -0.81 & -0.01 \\
\hline 10 & 0.2 & 0.1 & 0.05 & 0.25 & -0.16 & -0.24 & -0.02 & -0.35 & 0.47 & -0.02 & -0.02 & -0.01 & -0.33 & 0.52 & -0.02 & -0.02 & -0.02 & -0.34 & 0.02 & -0.02 & -0.02 & -0.02 & -0.35 & 0.02 \\
\hline 10 & 0.4 & 0.1 & 0.05 & 0.05 & 0.48 & 0.03 & 0.50 & -0.19 & 0.53 & 0.46 & -0.02 & 0.49 & -0.45 & 0.53 & 0.05 & 0.00 & 0.49 & -0.37 & 0.52 & -0.01 & -0.01 & 0.47 & -0.25 & 0.53 \\
\hline 10 & 0.4 & 0.1 & 0.01 & 0.15 & -0.19 & -0.59 & 0.00 & -0.80 & 0.05 & -0.02 & -0.48 & -0.01 & -0.81 & 0.18 & -0.02 & -0.02 & -0.02 & -0.49 & -0.01 & -0.02 & -0.02 & -0.01 & -0.52 & -0.01 \\
\hline
\end{tabular}

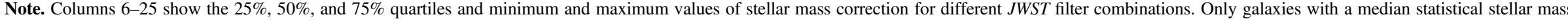

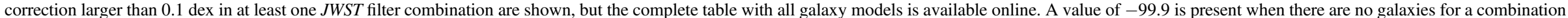
of output galaxy properties.

(This table is available in its entirety in machine-readable form.) 
Table 4

Distribution of the Statistical Stellar Mass Corrections $\Delta\left(\log _{10}\left(M^{*}\right)\right)=\log _{10}\left(M_{\text {out }}^{*} / M_{\odot}\right)-\log _{10}\left(M_{\text {in }}^{*} / M_{\odot}\right)$ for Different Output Galaxy Property Combinations Using Only Eight NIRCam Broad Bands

\begin{tabular}{|c|c|c|c|c|c|c|c|c|c|c|c|c|c|c|c|c|c|}
\hline \multirow{2}{*}{$\langle z\rangle$} & \multirow{2}{*}{$\begin{array}{c}Z \\
\left(Z_{\odot}\right)\end{array}$} & \multirow{2}{*}{$f_{\text {cov }}$} & \multirow{2}{*}{$\begin{array}{l}\text { SFH } \\
\text { (Gyr) }\end{array}$} & \multirow{2}{*}{$\begin{array}{c}t \\
(\mathrm{Gyr})\end{array}$} & \multirow{2}{*}{$\begin{array}{c}\langle E(B-V)\rangle \\
(\mathrm{mag})\end{array}$} & \multirow{2}{*}{$\mathrm{S} / \mathrm{N}_{\mathrm{F} 150 \mathrm{~W}}$} & \multicolumn{11}{|c|}{$P\left(\Delta\left(\log _{10}\left(M^{*}\right)\right)\right)$} \\
\hline & & & & & & & -1.075 & -1.025 & -0.975 & -0.925 & -0.875 & $\cdots$ & 0.875 & 0.925 & 0.975 & 1.025 & 1.075 \\
\hline 7 & 0.2 & 0 & 0.01 & 0.01 & 0.05 & 10 & 0.00 & 0.00 & 0.00 & 0.00 & 0.00 & $\ldots$ & 0.00 & 0.00 & 0.00 & 0.00 & 0.00 \\
\hline 7 & 0.2 & 0 & 0.1 & 0.05 & 0.15 & 10 & 0.00 & 0.00 & 0.00 & 0.00 & 0.00 & $\ldots$ & 0.00 & 0.01 & 0.00 & 0.00 & 0.00 \\
\hline 7 & 0.04 & 1 & 0.01 & 0.01 & 0.25 & 10 & 0.07 & 0.00 & 0.20 & 0.00 & 0.67 & $\ldots$ & 0.00 & 0.00 & 0.00 & 0.00 & 0.00 \\
\hline
\end{tabular}

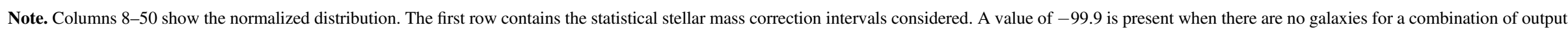
galaxy properties. The complete table is available online.

(This table is available in its entirety in machine-readable form.) 
Table 5

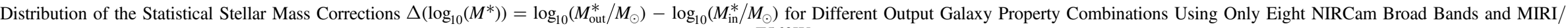
F560W

\begin{tabular}{|c|c|c|c|c|c|c|c|c|c|c|c|c|c|c|c|c|c|}
\hline \multirow{2}{*}{$\langle z\rangle$} & \multirow{2}{*}{$\begin{array}{c}Z \\
\left(Z_{\odot}\right)\end{array}$} & \multirow{2}{*}{$f_{\text {cov }}$} & \multirow{2}{*}{$\begin{array}{l}\text { SFH } \\
\text { (Gyr) }\end{array}$} & \multirow{2}{*}{$\begin{array}{c}t \\
(\mathrm{Gyr})\end{array}$} & \multirow{2}{*}{$\begin{array}{c}\langle E(B-V)\rangle \\
(\mathrm{mag})\end{array}$} & \multirow{2}{*}{$\mathrm{S} / \mathrm{N}_{\mathrm{F} 150 \mathrm{~W}}$} & \multicolumn{11}{|c|}{$P\left(\Delta\left(\log _{10}\left(M^{*}\right)\right)\right)$} \\
\hline & & & & & & & -1.075 & -1.025 & -0.975 & -0.925 & -0.875 & $\cdots$ & 0.875 & 0.925 & 0.975 & 1.025 & 1.075 \\
\hline 7 & 0.2 & 0 & 0.01 & 0.01 & 0.05 & 10 & 0.00 & 0.00 & 0.00 & 0.00 & 0.00 & $\ldots$ & 0.00 & 0.00 & 0.00 & 0.00 & 0.00 \\
\hline 7 & 0.2 & 0 & 0.1 & 0.01 & 0.15 & 10 & 0.00 & 0.00 & 0.00 & 0.00 & 0.01 & $\cdots$ & 0.00 & 0.00 & 0.00 & 0.00 & 0.00 \\
\hline 7 & 0.2 & 0 & 0.01 & 0.01 & 0.25 & 10 & 0.01 & 0.00 & 0.00 & 0.00 & 0.00 & $\ldots$ & 0.00 & 0.00 & 0.00 & 0.00 & 0.00 \\
\hline
\end{tabular}

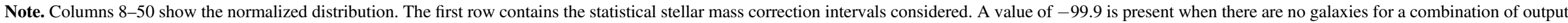
galaxy properties. The complete table is available online.

(This table is available in its entirety in machine-readable form.) 
Table 6

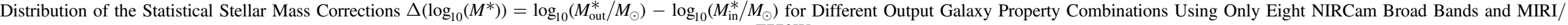
F770W

\begin{tabular}{|c|c|c|c|c|c|c|c|c|c|c|c|c|c|c|c|c|c|}
\hline \multirow{2}{*}{$\langle z\rangle$} & \multirow{2}{*}{$\begin{array}{c}Z \\
\left(Z_{\odot}\right)\end{array}$} & \multirow{2}{*}{$f_{\text {cov }}$} & \multirow{2}{*}{$\begin{array}{l}\text { SFH } \\
\text { (Gyr) }\end{array}$} & \multirow{2}{*}{$\begin{array}{c}t \\
(\mathrm{Gyr})\end{array}$} & \multirow{2}{*}{$\begin{array}{c}\langle E(B-V)\rangle \\
(\mathrm{mag})\end{array}$} & \multirow{2}{*}{$\mathrm{S} / \mathrm{N}_{\mathrm{F} 150 \mathrm{~W}}$} & \multicolumn{11}{|c|}{$P\left(\Delta\left(\log _{10}\left(M^{*}\right)\right)\right)$} \\
\hline & & & & & & & -1.075 & -1.025 & -0.975 & -0.925 & -0.875 & $\cdots$ & 0.875 & 0.925 & 0.975 & 1.025 & 1.075 \\
\hline 7 & 0.2 & 0 & 0.01 & 0.01 & 0.05 & 10 & 0.00 & 0.00 & 0.00 & 0.00 & 0.00 & $\ldots$ & 0.00 & 0.00 & 0.00 & 0.00 & 0.00 \\
\hline 7 & 0.2 & 1 & 0.1 & 0.05 & 0.05 & 10 & 0.00 & 0.00 & 0.00 & 0.00 & 0.00 & $\cdots$ & 0.00 & 0.01 & 0.01 & 0.00 & 0.00 \\
\hline 7 & 0.2 & 0 & 0.01 & 0.01 & 0.25 & 10 & 0.01 & 0.00 & 0.01 & 0.00 & 0.00 & $\ldots$ & 0.00 & 0.00 & 0.00 & 0.00 & 0.00 \\
\hline
\end{tabular}

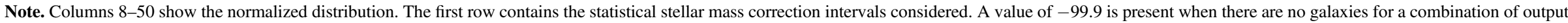
galaxy properties. The complete table is available online.

(This table is available in its entirety in machine-readable form.) 
Table 7

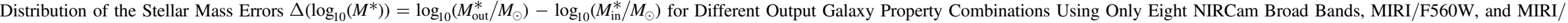
F770W

\begin{tabular}{|c|c|c|c|c|c|c|c|c|c|c|c|c|c|c|c|c|c|}
\hline \multirow{3}{*}{$\langle z\rangle$} & \multirow{3}{*}{$\begin{array}{c}Z \\
\left(Z_{\odot}\right)\end{array}$} & \multirow{3}{*}{$f_{\text {cov }}$} & \multirow{3}{*}{$\begin{array}{l}\text { SFH } \\
\text { (Gyr) }\end{array}$} & \multirow{3}{*}{$\begin{array}{c}t \\
(\mathrm{Gyr})\end{array}$} & \multirow{3}{*}{$\begin{array}{c}\langle E(B-V)\rangle \\
(\mathrm{mag})\end{array}$} & \multirow{3}{*}{$\mathrm{S} / \mathrm{N}_{\mathrm{F} 150 \mathrm{~W}}$} & \multicolumn{11}{|c|}{$P\left(\Delta\left(\log _{10}\left(M^{*}\right)\right)\right)$} \\
\hline & & & & & & & & & & & & & & & & & \\
\hline & & & & & & & $-1.0 / 5$ & -1.025 & -0.915 & -0.925 & -0.875 & $\cdots$ & 0.875 & 0.925 & 0.975 & 1.025 & 1.075 \\
\hline 7 & 0.2 & 0 & 0.01 & 0.01 & 0.05 & 10 & 0.00 & 0.00 & 0.00 & 0.00 & 0.00 & $\ldots$ & 0.00 & 0.00 & 0.00 & 0.00 & 0.00 \\
\hline 7 & 0.2 & 1 & 0.1 & 0.05 & 0.05 & 10 & 0.00 & 0.00 & 0.00 & 0.00 & 0.00 & $\ldots$ & 0.00 & 0.01 & 0.01 & 0.00 & 0.00 \\
\hline 7 & 0.2 & 0 & 0.01 & 0.01 & 0.25 & 10 & 0.01 & 0.00 & 0.01 & 0.00 & 0.00 & $\ldots$ & 0.00 & 0.00 & 0.00 & 0.00 & 0.00 \\
\hline
\end{tabular}

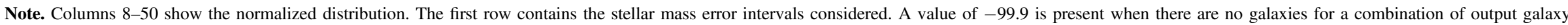
properties. The complete table is available online.

(This table is available in its entirety in machine-readable form.) 


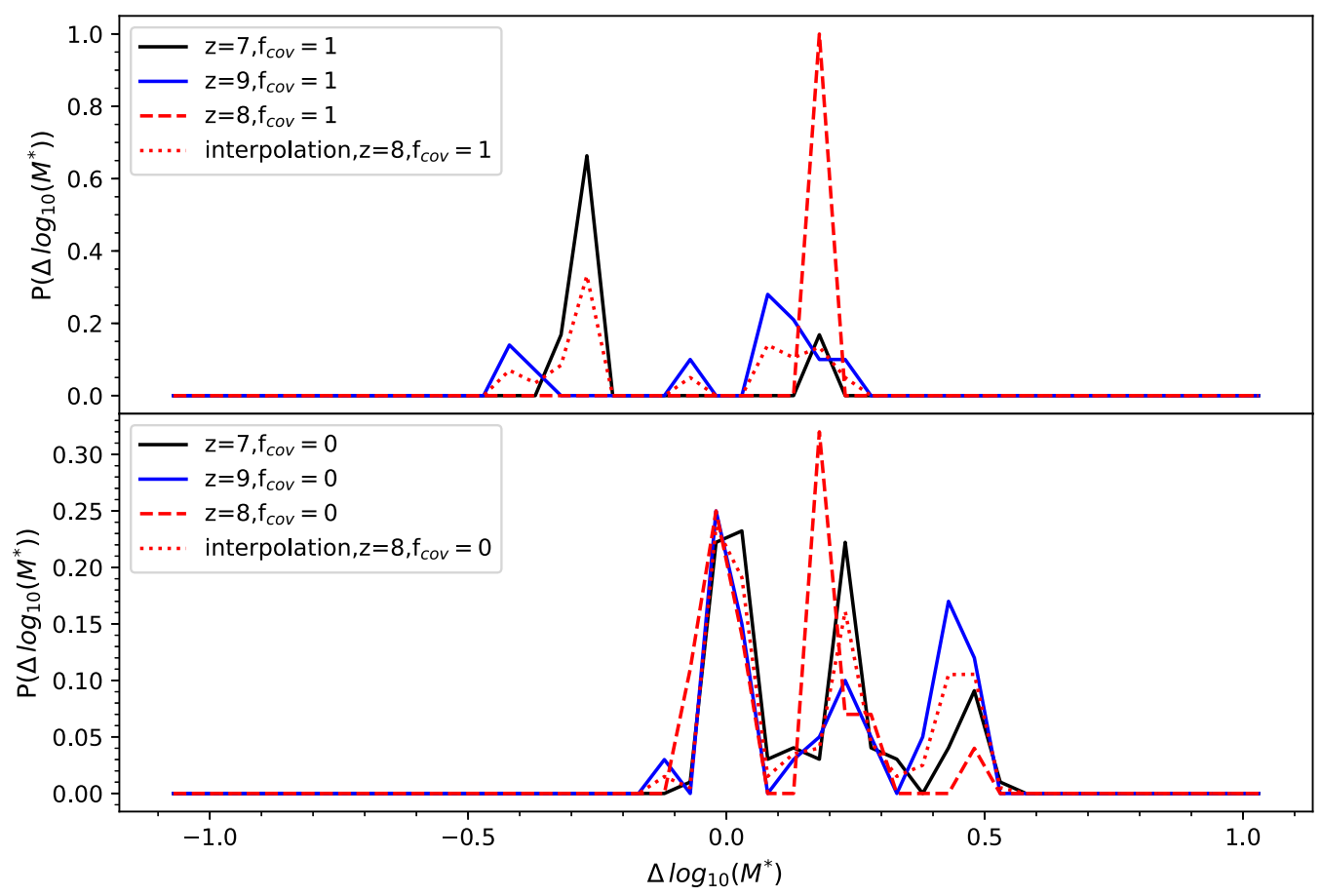

Figure 7. Example of a distribution of the statistical stellar mass corrections for three SED templates with the same parameter values, except the redshift. We show the distribution for a template at $z=7$ (solid black line), $z=8$ (dashed red line), and $z=9$ (solid blue line) and the interpolation between the distribution for the template at $z=7$ and 9 (dotted red line). We show two examples, i.e., for templates with $f_{\text {cov }}=1$ (top) and $f_{\text {cov }}=0$ (bottom).

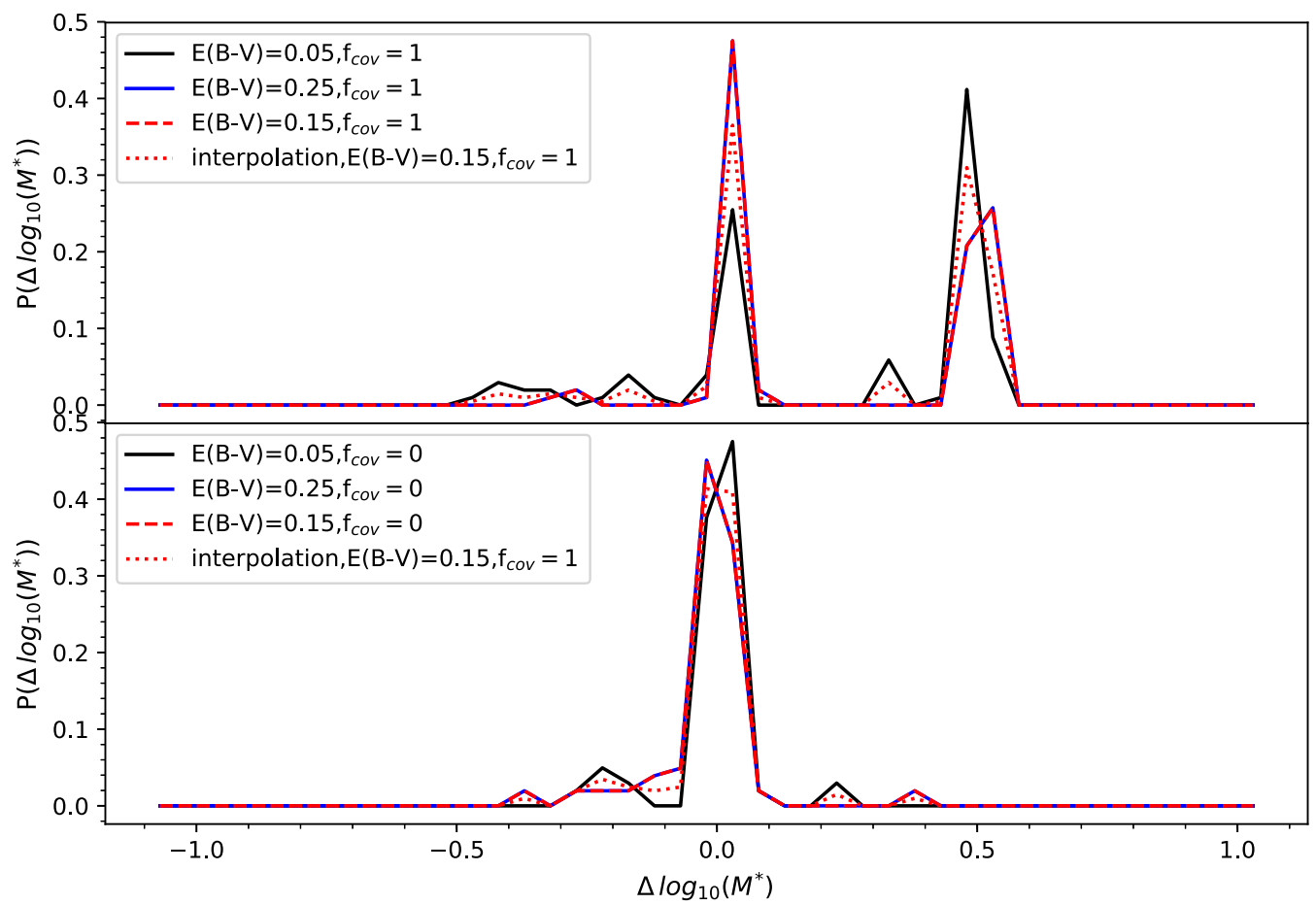

Figure 8. Example of a distribution of the statistical stellar mass corrections for three SED templates with the same parameter values, except the color excess. We show the distribution for a template with $E(B-V)=0.05 \mathrm{mag}$ (solid black line), $E(B-V)=0.15 \mathrm{mag}$ (dashed red line), and $E(B-V)=0.25 \mathrm{mag}$ (solid blue line) and the interpolation between the distribution for the template with $E(B-V)=0.05$ and $0.25 \mathrm{mag}$ (dotted red line). We show two examples, i.e., for templates with $f_{\text {cov }}=1$ (top) and $f_{\text {cov }}=0$ (bottom).

before and after applying the statistical errors. Excluding $z=7$, the mean absolute error on the stellar mass distributions decreases by $20 \%-50 \%$ after applying the stellar mass corrections derived here. It is evident that, for this specific case, the main improvement on applying the statistical stellar mass correction consists of not overestimating the number of extremely low stellar mass objects $\left(M^{*}<10^{7} M_{\odot}\right)$ that are indeed expected to be an important fraction of the galaxy population that will be observed with JWST at $z>7$ (Ceverino et al. 2019). This may be an important issue for future works 


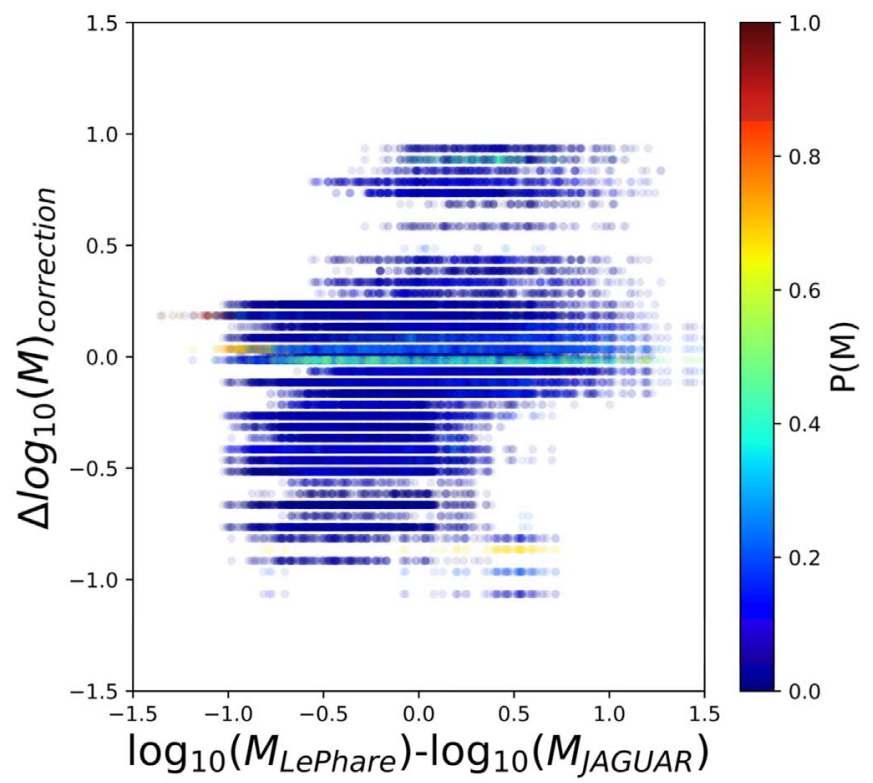

Figure 9. Comparison between the stellar mass correction and the difference between the JAGUAR stellar mass and the stellar mass recovered with LePhare. We color-coded each correction by its probability (Table 4).

that aim to recover the proper value of the stellar mass function faint-end slope at high- $z$.

As already mentioned, the corrections derived in this work are not exhaustive. Indeed, an additional offset between the original stellar mass and the one derived with the Yggdrasil templates is present, and it is due to different emission-line equivalent-width recipes and SFHs used in Yggdrasil and BEAGLE.

Overall, the statistical stellar mass corrections improve the stellar mass distribution derivation obtained with the Yggdrasil templates, even if the galaxies have completely different SFHs and emission-line recipes.

\section{Conclusions}

In a series of papers, of which this is the third, we have created and analyzed a mock galaxy sample to investigate how different galaxy properties will be derived using the JWST NIRCam broadband filters and the two MIRI filters F560W and F770W for galaxies at redshifts between 7 and 10. In particular, we consider a mock galaxy sample with good photometry $\left(\mathrm{S} / \mathrm{N}_{\mathrm{F} 150 \mathrm{w}}>10\right)$ for which the redshift is well recovered, and we derive galaxy properties fitting the broadband SED in a similar way to what will be done for galaxies in future JWST deep blank-field imaging surveys. Then, we compare the derived galaxy properties with the input ones to understand the biases in the galaxy property estimation.

In this paper, we focus on the stellar mass estimation, providing a list of statistical stellar mass corrections to take into account when estimating stellar masses of large samples of high- $z$ galaxies that will be observed in the near future with JWST. These corrections can be used directly when stellar masses are derived using the LePhare code and the SED template considered here, and they can be generally considered as an indication of the possible template degeneracies affecting the stellar mass derivation. In particular, we provide the $25 \%, 50 \%$, and $75 \%$ quartiles and minimum and maximum value of the stellar mass offsets, together with the full stellar mass offset distributions, for each combination of output galaxy parameters and different JWST broadband filter combinations.

Median stellar mass offsets are generally small, but the $25 \%$ and $75 \%$ quartiles for some specific templates range from -0.83 to $0.88 \mathrm{dex}$; therefore, some galaxy models may need significant stellar mass corrections.

In particular, we notice the following.

1. Galaxies without nebular emission lines in output that originally had emission lines have overestimated $\mathrm{SFH}$ and age. This results in an overestimation of the stellar mass that is even 10 times the original stellar mass. On the other hand, galaxies with nebular emission lines in output that were originally without emission lines have stellar masses that are generally underestimated, even down to 10 times the original mass.

2. The stellar mass of galaxies is more difficult to estimate at $z=10$, when only the eight NIRCam bands are considered, but also at $z=7$. The first effect is due to the fact that no NIRCam bands purely cover the $\lambda>4000 \AA$ break; therefore, MIRI bands are necessary to trace these wavelengths and improve the stellar mass estimation. The reduced age-extinction degeneracy is instead one of the reasons why the stellar mass recovery is less difficult at $z>7$ than at $z=7$.

3. Stellar masses are well recovered for galaxies with output color excess between 0.2 and $0.3 \mathrm{mag}$, i.e., the maximum value analyzed in this paper, while the stellar mass estimation is less accurate for relatively dust-free templates.

4. For galaxies without emission lines, i.e., quenched or star-forming galaxies with $f_{\text {cov }}=0$, the stellar mass is generally well recovered, except for the lowest metallicity considered, which is $1 / 50 Z_{\odot}$.

5. We apply the statistical stellar mass corrections presented in this work to the JAGUAR catalog, which has been derived using different assumptions (Williams et al. 2018). The discrepancies in the recovered stellar mass distribution decrease by $20 \%-50 \%$ at $z>7$ when including the stellar mass corrections. The results highlight the importance of the considered statistical stellar mass corrections to properly derive the distribution of the low-mass galaxy population that otherwise tends to be overestimated in number. This is indeed essential for future works that aim at deriving the faint-end slope of the high- $z$ stellar mass function.

Overall, the stellar mass estimation is challenging for young galaxies with nebular emission lines or galaxies with emission lines that have not been properly identified. Adding at least one of the two MIRI bands at the shortest wavelengths improves the stellar mass estimation, refining the average estimation or reducing the worst outliers.

In the future, additional investigations will be necessary to expand this analysis on extremely dusty objects and galaxies with a nonnegligible contribution by active galactic nuclei. 

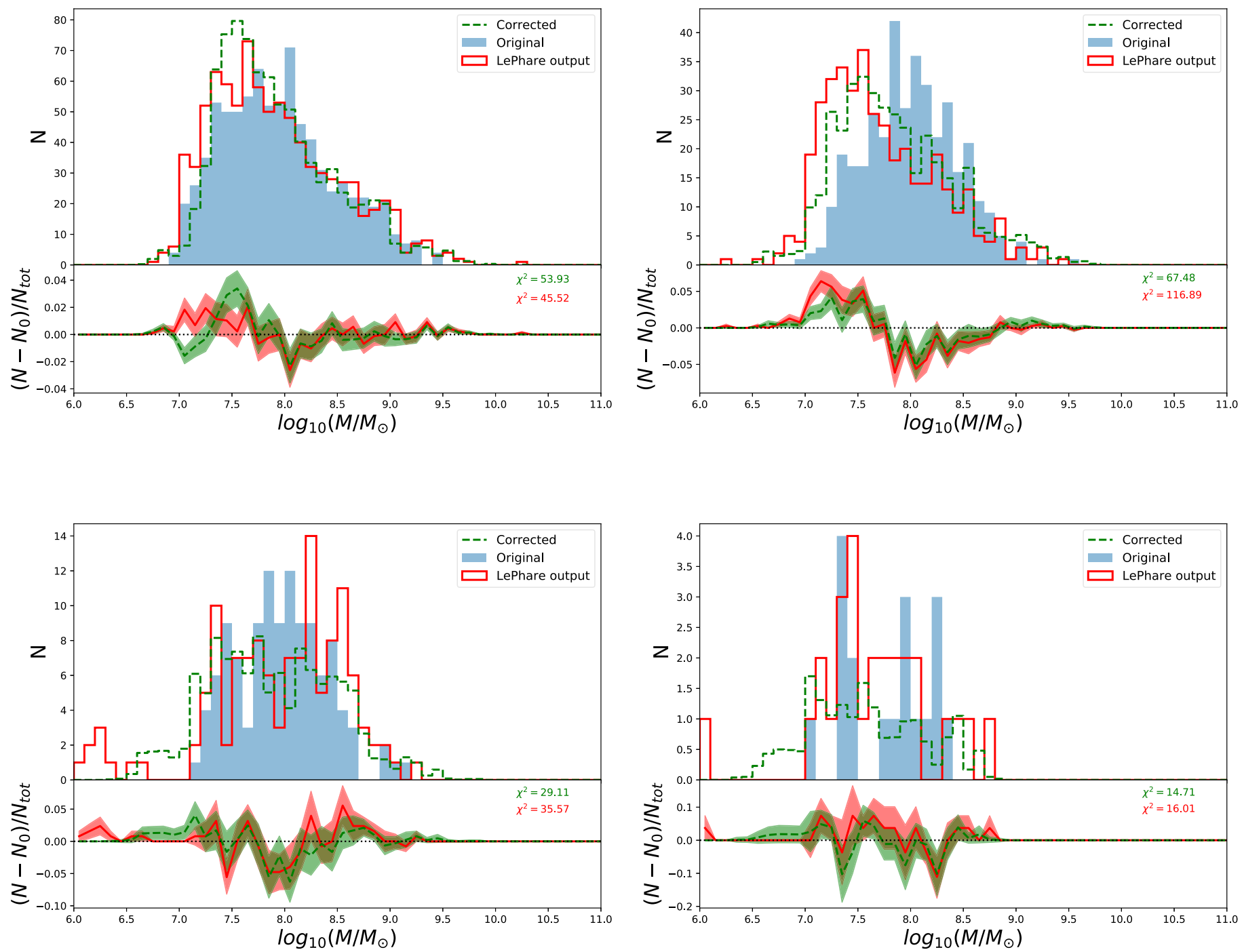

Figure 10. Top panels: stellar mass distributions in the JAGUAR catalog (filled blue histogram) and derived with the Yggdrasil templates before (red line) and after (dashed green line) the statistical stellar mass correction. Bottom panels: offsets between the original JAGUAR stellar mass and the stellar mass derived with the Yggdrasil templates before (red line) and after (dashed green line) the statistical stellar mass correction. The average values of the absolute number differences are shown on the right. Shaded areas show the statistical uncertainties. Both stellar mass distributions and offsets are shown for four redshift bins: $6.5 \leqslant z<7.5$ (top left), $7.5 \leqslant z<8.5$ (top right), $8.5 \leqslant z<9.5$ (bottom left), and $9.5 \leqslant z<10.5$ (bottom right).

L.B. and K.I.C. acknowledge funding from the European Research Council through the award of Consolidator Grant ID 681627-BUILDUP. P.G.P.-P.G. acknowledges support from Spanish Government grant AYA2015-63650-P. L.C. acknowledges support by the Spanish Ministry for Science, Innovation and Universities under project ESP2017-83197.

Software: Yggdrasil (Zackrisson et al. 2011), LePhare (Arnouts et al. 1999; Ilbert et al. 2006).

\section{ORCID iDs}

L. Bisigello (1) https://orcid.org/0000-0003-0492-4924

K. I. Caputi (1) https://orcid.org/0000-0001-8183-1460

P. G. Pérez-González (1) https://orcid.org/0000-00034528-5639

A. Koekemoer (1) https://orcid.org/0000-0002-6610-2048

N. Grogin (1) https://orcid.org/0000-0001-9440-8872

P. van der Werf (i) https://orcid.org/0000-0001-5434-5942

\section{References}

Arnouts, S., Cristiani, S., Moscardini, L., et al. 1999, MNRAS, 310, 540 Bisigello, L., Caputi, K. I., Colina, L., et al. 2016, ApJS, 227, 19 Bisigello, L., Caputi, K. I., Colina, L., et al. 2017, ApJS, 231, 3 Bisigello, L., Caputi, K. I., Grogin, N., \& Koekemoer, A. 2018, A\&A, 609, A82

Boogaard, L. A., Brinchmann, J., Bouché, N., et al. 2018, A\&A, 619, 27 Brinchmann, J., Charlot, S., White, S. D. M., et al. 2004, MNRAS, 351, 1151 Bruzual, G., \& Charlot, S. 2003, MNRAS, 344, 1000

Calzetti, D., Armus, L., Bohlin, R. C., et al. 2000, ApJ, 533, 682

Caputi, K. I., Deshmukh, S., Ashby, M. L. N., et al. 2017, ApJ, 849, 45 Caputi, K. I., Ilbert, O., Laigle, C., et al. 2015, ApJ, 810, 73

Ceverino, D., Klessen, R. S., \& Glover, S. C. O. 2019, MNRAS, 484, 1366 Chevallard, J., \& Charlot, S. 2016, MNRAS, 462, 1415

Erb, D. K., Shapley, A. E., Pettini, M., et al. 2006, ApJ, 644, 813 Gardner, J. P., Mather, J. C., Clampin, M., et al. 2009, ITASS, 10, 1 Ilbert, O., Arnouts, S., McCracken, H. J., et al. 2006, A\&A, 457, 841 Kroupa, P. 2002, Sci, 295, 82

Maier, C., Ziegler, B. L., Lilly, S. J., et al. 2015, A\&A, 577, A14

Maiolino, R., Nagao, T., Grazian, A., et al. 2008, A\&A, 488, 463 
Noeske, K. G., Weiner, B. J., Faber, S. M., et al. 2007, ApJL, 660, L43

Oke, J. B., \& Gunn, J. E. 1983, ApJ, 266, 713

Peng, Y.-j., Lilly, S. J., Kovač, K., et al. 2010, ApJ, 721, 193

Rieke, G. H., Wright, G. S., Böker, T., et al. 2015, PASP, 127, 584

Rieke, M. J., Kelly, D., \& Horner, S. 2005, Proc. SPIE, 5904, 1

Rodighiero, G., Daddi, E., Baronchelli, I., et al. 2011, ApJL, 739, L40

Santini, P., Ferguson, H. C., Fontana, A., et al. 2015, ApJ, 801, 97

Stark, D. P., Schenker, M. A., Ellis, R., et al. 2013, ApJ, 763, 129
Tasca, L. A. M., Le Fèvre, O., Hathi, N. P., et al. 2015, A\&A, 581, A54

Tremonti, C. A., Heckman, T. M., Kauffmann, G., et al. 2004, ApJ, 613, 898

Whitaker, K. E., Franx, M., Leja, J., et al. 2014, ApJ, 795, 104

Williams, C. C., Curtis-Lake, E., Hainline, K. N., et al. 2018, ApJS, 236, 33

Wright, G. S., Wright, D., Goodson, G. B., et al. 2015, PASP, 127, 595

Zackrisson, E., Rydberg, C.-E., Schaerer, D., Östlin, G., \& Tuli, M. 2011, ApJ, 740,13 